\title{
CELLULARITY OF GENERALIZED SCHUR ALGEBRAS VIA CAUCHY DECOMPOSITION
}

\author{
JONATHAN D. AXTELL
}

\begin{abstract}
We describe a generalization of Hashimoto and Kurano's Cauchy filtration for divided powers algebras. This filtration is then used to provide a cellular structure for generalized Schur algebras associated to an arbitrary cellular algebra, $A$. Applications to the cellularity of wreath product algebras $A \prec \mathfrak{S}_{d}$ are also considered.
\end{abstract}

\section{INTRODUCTION}

Let $\mathbb{k}$ be a noetherian integral domain and suppose $A$ is a cellular $\mathbb{k}$-algebra [8]. Then Geetha and Goodman [7] showed that the wreath product algebra

$$
A \text { 乙 } \mathfrak{S}_{d}=A^{\otimes d} \rtimes \mathbb{k} \mathfrak{S}_{d}
$$

is cellular, provided that all of the cell ideals of $A$ are cyclic. On the other hand, the generalized Schur algebras $S^{A}(n, d)$ were defined by Evseev and Kleshchev $[4,5]$ in order to prove the Turner double conjecture. These algebras are related to wreath product algebras by a generalized Schur-Weyl duality established in [4].

In this paper, we describe a cellular structure for the generalized Schur algebra $S^{A}(n, d)$ for an arbitrary cellular algebra $A$ and for all integers $n, d \geq 0$. This extends some results of Kleshchev and Muth [13, 14, 15]. It follows, for example, from results of [15] that the algebra $S^{A}(n, d)$ is cellular for certain algebras $A$ which are both cellular and quasi-hereditary. We note that for such algebras, the cell ideals are automatically cyclic. The method used in this paper, however, does not require any additional assumptions on the cellular algebra.

Our approach is motivated by that of [17], where Krause used the Cauchy decomposition of divided powers $[1,12]$ to describe the highest weight structure of categories of strict polynomial functors. As Krause mentions, this leads to an alternate proof of the fact that classical Schur algebras $S^{\mathbb{k}}(n, d)$ are quasi-hereditary, which follows by a Morita equivalence. As we will see, this approach can similarly be used to describe cellular structure.

We begin by constructing a generalized Cauchy filtration for the divided powers $\Gamma^{d} J$ of a given $\mathbb{k}$-module, $J$, which we assume is equipped with a filtration

$$
0=J_{1} \subset \cdots \subset J_{r}=J
$$

such that $J_{j} / J_{j-1} \cong U_{j} \otimes_{\mathbb{k}} V_{j}$, for some free $\mathbb{k}$-modules $U_{j}, V_{j}$ of finite rank. Our first main result is a generalized Cauchy decomposition formula (Theorem 5.14), which provides a filtration of $\Gamma^{d} J$ such that the associated graded object is a direct sum of modules of the form

$$
\bigoplus_{\boldsymbol{\lambda} \in \boldsymbol{\Lambda}} \mathcal{U}_{\boldsymbol{\lambda}} \otimes_{\mathbb{k}} v_{\boldsymbol{\lambda}}
$$

where $\mathcal{U}_{\boldsymbol{\lambda}}, \mathcal{V}_{\boldsymbol{\lambda}}$ are generalized Weyl modules defined in Section 5.6 and $\boldsymbol{\Lambda}$ denotes a set of $r$-multipartitions.

This paper was supported by the National Research Foundation of Korea (NRF) funded by the Ministry of Science (NRF-2017R1C1B5018384). 
The generalized Schur algebra $S^{A}(n, d)$ may be identified as the $d$-th divided power $\Gamma^{d} \mathrm{M}_{n}(A)$, where $\mathrm{M}_{n}(A)$ is the algebra of size $n$ matrices over $A$. We are thus able to use the above decomposition, together with König and Xi's characterization of cellular algebras in [16], to prove our second main result (Theorem 6.5) which shows that generalized Schur algebras are cellular. In Example 6.6, we describe a corresponding cellular basis explicitly for a particular case, $S^{Z}(1,2)$, where $Z$ is a zig-zag algebra (considered as an ordinary algebra rather than a superalgebra, as in $[15])$.

As a consequence of generalized Schur-Weyl duality, Corollary 6.8 shows that the wreath product algebras $A<\mathfrak{S}_{d}$ are cellular for an arbitrary cellular algebra $A$. This provides an alternate proof of the main result in [7], for the case where $A$ is cyclic cellular, and a more recent result of Green [11], for the general case where $A$ is an arbitrary cellular algebra.

\section{PRELiminaries}

Assume throughout that $\mathbb{k}$ is a commutative ring, unless mentioned otherwise. The notation $\sharp$ is used for the cardinality of a set.

2.1. Weights, partitions, and sequences. Write $\mathbb{N}$ and $\mathbb{N}_{0}$ to denote the sets of positive and nonnegative integers, respectively, with the usual total order. More generally, suppose that $\mathcal{B}$ is a countable totally ordered set which is bounded below. Any elements $a, b \in \mathcal{B}$ determine an interval

$$
[a, b]:=\{c \in \mathcal{B} \mid a \leq c \leq b\}
$$

which is empty unless $a \leq b$.

A weight $($ on $\mathcal{B})$ is a sequence of nonnegative integers $\mu=\left(\mu_{b}\right)_{b \in \mathcal{B}}$ such that $\mu_{b}=0$ for almost all $b$. Let $\Lambda(\mathcal{B})$ denote the set of all weights on $\mathcal{B}$. A partition (on $\mathcal{B}$ ) is a weight $\lambda \in \Lambda(\mathcal{B})$ such that

$$
b<c \text { implies } \lambda_{b} \geq \lambda_{c},{ }^{\forall} b, c \in \mathcal{B} \text {. }
$$

The subset of partitions is denoted $\Lambda^{+}(\mathcal{B}) \subset \Lambda(\mathcal{B})$. The size of a weight $\mu$ is the integer $|\mu|:=\sum_{b} \mu_{b}$. Let $\Lambda_{d}(\mathcal{B})$ denote the set of all weights of size $d$ and write

$$
\Lambda_{d}^{+}(\mathcal{B}):=\Lambda^{+}(\mathcal{B}) \cap \Lambda_{d}(\mathcal{B})
$$

for each $d \in \mathbb{N}_{0}$.

Remark 2.1. In this notation and elsewhere, we will use the convention of replacing an argument of the form $[1, n]$ by " $n$ " for any $n \in \mathbb{N}_{0}$, so that for example $\Lambda(n)$ denotes the set $\Lambda([1, n])$ of weights of the form $\mu=\left(\mu_{1}, \ldots, \mu_{n}\right)$.

We also identify each set $\Lambda(n)$ as a subset of $\Lambda(\mathbb{N})$ in the obvious way and write

$$
l(\mu):=\min \left\{n \in \mathbb{N}_{0} \mid \mu \in \Lambda(n)\right\}
$$

to denote the length of a weight $\mu \in \Lambda(\mathbb{N})$. For example, the length $l(\lambda)$ of a partition $\lambda=\left(\lambda_{1}, \lambda_{2}, \ldots\right)$ in $\Lambda^{+}(\mathbb{N})$ equals the number of positive parts, $\lambda_{i} \in \mathbb{N}$.

Definition 2.2. Let $d \in \mathbb{N}_{0}$. Recall that the lexicographic ordering on $\Lambda_{d}(\mathbb{N})$ is the total order defined by setting $\lambda \leq \mu$ if $\lambda_{j} \leq \mu_{j}$ whenever $\lambda_{i}=\mu_{i}$ for all $i<j$. We use the notation $\preceq$ to denote the restriction of $\leq$ to the subset $\Lambda_{d}^{+}(\mathbb{N})$ of partitions of size $d$.

Now fix $d \in \mathbb{N}$, and write $\operatorname{seq}^{d}(\mathcal{B})$ to denote the set of all functions

$$
\mathrm{b}:[1, d] \rightarrow \mathcal{B} \text {. }
$$


We identify $\operatorname{seq}^{d}(\mathcal{B})$ with $\mathcal{B}^{d}$ by setting $\mathrm{b}=\left(b_{1}, \ldots, b_{d}\right)$, with $b_{i}=\mathrm{b}(i)$ for all $i \in[1, d]$. The symmetric group $\mathfrak{S}_{d}$ of permutations of $[1, d]$ acts on $\operatorname{seq}^{d}(\mathcal{B})$ from the right via composition. We write $\mathrm{b} \sim \mathrm{c}$ if there exists $\sigma \in \mathfrak{S}_{d}$ with $\mathrm{c}=\mathrm{b} \sigma$.

The weight of a sequence $\mathrm{b} \in \operatorname{seq}^{d}(\mathcal{B})$ is the element of $\Lambda_{d}(\mathcal{B})$ defined by

$$
\mu(\mathrm{b}):=\left(\mu_{c}\right)_{c \in \mathcal{B}}, \quad \text { where } \quad \mu_{c}=\sharp\left\{i \mid b_{i}=c\right\} \quad{ }^{\forall} c \in \mathcal{B} .
$$

We note the following elementary result.

Lemma 2.3. The map $\mu: \operatorname{seq}^{d}(\mathcal{B}) \rightarrow \Lambda_{d}(\mathcal{B})$, sending $\mathrm{b} \mapsto \mu(\mathrm{b})$, induces a bijection: $\operatorname{seq}^{d}(\mathcal{B}) / \mathfrak{S}_{d} \simeq \Lambda_{d}(\mathcal{B})$.

Proof. We may assume that $\mathcal{B}$ is nonempty. Since $\mathcal{B}$ is bounded below, it is possible to write the elements explicity in the form

$$
\mathcal{B}=\left\{b_{1}^{\mathcal{B}}<b_{2}^{\mathcal{B}}<\ldots\right\} .
$$

To show that the map $\mathrm{b} \mapsto \mu(\mathrm{b})$ is surjective, note that a right inverse is given by

$$
\Lambda_{d}(\mathcal{B}) \rightarrow \operatorname{seq}^{d}(\mathcal{B}): \mu \mapsto \mathrm{b}_{\mu}:=\left(b_{1}^{\mathcal{B}}, \ldots, b_{1}^{\mathcal{B}}, b_{2}^{\mathcal{B}}, \ldots, b_{2}^{\mathcal{B}}, \ldots\right)
$$

where $b_{1}^{\mathcal{B}}$ occurs with multiplicity $\mu_{b_{1}^{\mathcal{B}}}$, etc. Finally, it is easy to see that $\mathrm{b} \sim \mathrm{c}$ if and only if $\mu(\mathrm{b})=\mu(\mathrm{c})$, which completes the proof.

Suppose more generally that $\mathcal{B}_{1}, \ldots, \mathcal{B}_{r}$ is a collection of bounded below, totally ordered sets. We again consider the product $\mathcal{B}=\mathcal{B}_{1} \times \cdots \times \mathcal{B}_{r}$ as a bounded below, totally ordered set via the lexicographic ordering.

The symmetric group $\mathfrak{S}_{d}$ acts diagonally on the following product

$$
\operatorname{seq}^{d}\left(\mathcal{B}_{1}, \ldots, \mathcal{B}_{r}\right):=\operatorname{seq}^{d}\left(\mathcal{B}_{1}\right) \times \cdots \times \operatorname{seq}^{d}\left(\mathcal{B}_{r}\right) .
$$

Notice that the bijection

$$
\theta: \operatorname{seq}^{d}\left(\mathcal{B}_{1}, \ldots, \mathcal{B}_{r}\right) \simeq \operatorname{seq}^{d}(\mathcal{B})
$$

defined by

$$
\theta\left(\mathrm{b}^{(1)}, \ldots, \mathrm{b}^{(r)}\right): i \mapsto\left(b_{i}^{(1)}, \ldots, b_{i}^{(r)}\right), \quad{ }^{\forall} i \in[1, d],
$$

is $\mathfrak{S}_{d}$-equivariant. It thus follows as an immediate consequence of Lemma 2.3 that there is a bijection

$$
\operatorname{seq}^{d}\left(\mathcal{B}_{1}, \ldots, \mathcal{B}_{r}\right) / \mathfrak{S}_{d} \simeq \Lambda_{d}(\mathcal{B})
$$

where $\operatorname{seq}^{d}\left(\mathcal{B}_{1}, \ldots, \mathcal{B}_{r}\right) / \mathfrak{S}_{d}$ denotes the set of diagonal $\mathfrak{S}_{d}$-orbits.

2.2. Multipartitions. Suppose $d \in \mathbb{N}_{0}$ and let $\mathcal{B}_{1}, \ldots, \mathcal{B}_{r}$ be as above. Then we use the following notation for the product

$$
\Lambda^{+}\left(\mathcal{B}_{1}, \ldots, \mathcal{B}_{r}\right):=\Lambda^{+}\left(\mathcal{B}_{1}\right) \times \cdots \times \Lambda^{+}\left(\mathcal{B}_{r}\right) .
$$

whose elements are called $r$-multipartions and denoted $\boldsymbol{\lambda}=\left(\lambda^{(1)}, \ldots, \lambda^{(r)}\right)$. The weight of an $r$-multipartion $\boldsymbol{\lambda}$ is the element of $\Lambda(r)$ defined by

$$
|\boldsymbol{\lambda}|:=\left(\left|\lambda^{(1)}\right|, \ldots,\left|\lambda^{(r)}\right|\right) .
$$

We call $\|\boldsymbol{\lambda}\|:=\sum\left|\lambda^{(j)}\right|$ the total weight (or size) of $\boldsymbol{\lambda}$.

Given $\mu \in \Lambda(r)$ and $d \in \mathbb{N}_{0}$, we write

$$
\Lambda_{\mu}^{+}\left(\mathcal{B}_{1}, \ldots, \mathcal{B}_{r}\right):=\Lambda_{\mu_{1}}^{+}\left(\mathcal{B}_{1}\right) \times \cdots \times \Lambda_{\mu_{r}}^{+}\left(\mathcal{B}_{r}\right)
$$

and

$$
\Lambda_{d}^{+}\left(\mathcal{B}_{1}, \ldots, \mathcal{B}_{r}\right):=\bigsqcup_{\nu \in \Lambda_{d}(r)} \Lambda_{\nu}^{+}\left(\mathcal{B}_{1}, \ldots, \mathcal{B}_{r}\right)
$$

to denote the subset of $r$-multipartions of weight $\mu$, resp. total weight $d$.

In the special case where $\mathcal{B}_{j}=\mathbb{N}$ for $j \in[1, r]$, note that

$$
\Lambda^{+}(\mathbb{N}, \ldots, \mathbb{N})=\Lambda^{+}(\mathbb{N})^{r} \text {. }
$$


We then use the following notation

$$
\Lambda_{d}^{+}(\mathbb{N})^{r}:=\Lambda_{d}^{+}(\mathbb{N}, \ldots, \mathbb{N}), \quad \Lambda_{\mu}^{+}(\mathbb{N})^{r}:=\Lambda_{\mu}^{+}(\mathbb{N}, \ldots, \mathbb{N})
$$

for $d \in \mathbb{N}_{0}$ and $\mu \in \Lambda_{r}(d)$, respectively.

The next definition describes a total order on the set of $r$-multipartitions of a fixed total weight.

Definition 2.4. Suppose $d, r \in \mathbb{N}$. Then $\Lambda_{d}^{+}(\mathbb{N})^{r}$ has a total order $\preceq$ defined as follows. For $r$-multipartitions $\boldsymbol{\mu}, \boldsymbol{\lambda} \in \Lambda_{\nu}^{+}(\mathbb{N})$ of weight $\nu \in \Lambda_{d}(r)$, we set $\boldsymbol{\lambda} \preceq \boldsymbol{\mu}$ if

$$
\lambda^{(j)} \preceq \mu^{(j)} \text {, whenever } \lambda^{(i)}=\mu^{(i)} \text { for all } i<j \text {. }
$$

We then extend $\preceq$ to all of $\Lambda_{d}^{+}(\mathbb{N})^{r}$ by setting $\boldsymbol{\lambda} \prec \boldsymbol{\mu}$ whenever $|\boldsymbol{\lambda}|<|\boldsymbol{\mu}|$ in the lexicographic ordering on $\Lambda_{d}(r)$.

Suppose $n_{1}, \ldots, n_{r} \in \mathbb{N}_{0}$ and $d \in \mathbb{N}$. Recalling the notation from Remark 2.1, we identify the set of $r$-multipartions

$$
\Lambda^{+}\left(n_{1}, \ldots, n_{r}\right):=\Lambda^{+}\left(\left[1, n_{1}\right], \ldots,\left[1, n_{r}\right]\right)
$$

as a subset of $\Lambda^{+}(\mathbb{N})^{r}$ and view $\preceq$ as a total order on $\Lambda_{d}^{+}\left(n_{1}, \ldots, n_{r}\right)$ by restriction.

2.3. Finitely generated projective modules. Let $\mathcal{M}_{\mathbb{k}}$ denote the category of all $\mathbb{k}$-modules and $\mathbb{k}$-linear maps. The full subcategory of finitely generated projective $\mathbb{k}$-modules is denoted $\mathcal{P}_{\mathbb{k}}$.

Given $M, N \in \mathcal{M}_{\mathbb{k}}$, we write $M \otimes N=M \otimes_{\mathbb{k}} N$ and $\operatorname{Hom}(M, N)=\operatorname{Hom}_{\mathbb{k}}(M, N)$. Also write $\operatorname{End}(M)$ to denote the $\mathbb{k}$-algebra $\operatorname{Hom}(M, M)$. If $M \in \mathcal{P}_{\mathbb{k}}$, we let $M^{\vee}=$ $\operatorname{Hom}(M, \mathbb{k})$ denote the $\mathbb{k}$-linear dual. For any $M, M^{\prime}, N, N^{\prime} \in \mathcal{P}_{\mathbb{k}}$, there is an isomorphism

$$
\operatorname{Hom}\left(M \otimes N, M^{\prime} \otimes N^{\prime}\right) \cong \operatorname{Hom}\left(M, M^{\prime}\right) \otimes \operatorname{Hom}\left(N, N^{\prime}\right)
$$

which is natural with respect to composition.

2.4. Divided and symmetric powers. Let $d \in \mathbb{N}$. Given $M \in \mathcal{P}_{\mathbb{k}}$, there is a right action of the symmetric group $\mathfrak{S}_{d}$ on the tensor power $M^{\otimes d}$ given by permuting tensor factors. We define the $d$-th divided power of $M$ to be the invariant submodule

$$
\Gamma^{d} M:=\left(M^{\otimes d}\right)^{\mathfrak{S}_{d}} .
$$

Similarly, the coinvariant module is denoted

$$
\operatorname{Sym}_{d} M:=\left(M^{\otimes d}\right)_{\mathfrak{S}_{d}}
$$

and called the $d$-th symmetric power of $M$. It follows by definition that

$$
\Gamma^{d}(M)^{\vee} \cong \operatorname{Sym}_{d}\left(M^{\vee}\right) \text {. }
$$

We also set $\Gamma^{0} M=\operatorname{Sym}_{0} M=\mathbb{k}$.

Note that the isomorphism (2.4) is usually taken as the definition of $\Gamma^{d} M$ (cf. [1]), while we have used the equivalent definition from [17] in terms of symmetric tensors.

2.5. The divided powers algebra. The category $\mathcal{M}_{\mathbb{k}}$ (resp. $\mathcal{P}_{\mathbb{k}}$ ) is a symmetric monoidal category with symmetry isomorphism

$$
\text { tw }: M \otimes N \stackrel{\sim}{\rightarrow} N \otimes M
$$

defined by $x \otimes y \mapsto y \otimes x$, for all $x \in M, y \in N$.

Suppose $M \in \mathcal{P}_{\mathbb{k}}$. Then

$$
\Gamma(M):=\bigoplus_{d \in \mathbb{N}_{0}} \Gamma^{d} M
$$


is an ( $\mathbb{N}_{0}$-graded) commutative algebra called the divided powers algebra, with multiplication defined on homogeneous components via the shuffle product: for $x \in \Gamma^{d} M$ and $y \in \Gamma^{e} M$, define

$$
x * y:=\sum_{\sigma \in \mathfrak{S}_{d+e}^{d, e}}(x \otimes y) \sigma
$$

where $\mathfrak{S}_{d+e}^{d, e}$ is the quotient group $\mathfrak{S}_{d+e} / \mathfrak{S}_{d} \times \mathfrak{S}_{e}$. For example, we have $x^{\otimes d} * x^{\otimes e}=$ $\left(\begin{array}{c}d+e \\ d\end{array}\right) x^{\otimes(d+e)}$ for any $x \in M$.

There is also a comultiplication, $\Delta: \Gamma(M) \rightarrow \Gamma(M) \otimes \Gamma(M)$, which is the $\mathbb{N}_{0^{-}}$ homogenous map whose graded components

$$
\Delta: \Gamma^{d} M \rightarrow \Gamma^{d-c} M \otimes \Gamma^{c} M
$$

are defined as the inclusions

$$
\left(M^{\otimes d}\right)^{\mathfrak{S}_{d}} \hookrightarrow\left(M^{\otimes d}\right)^{\mathfrak{S}_{d-c} \times \mathfrak{S}_{c}}
$$

induced by the embeddings $\mathfrak{S}_{d-c} \times \mathfrak{S}_{c} \hookrightarrow \mathfrak{S}_{d}$, for $c \in[0, d]$. These maps, together with the unit, $\mathbb{k}=\Gamma^{0} M \hookrightarrow \Gamma(M)$, and the counit, $\Gamma(M) \rightarrow \Gamma^{0} M$ (projection onto degree 0$)$, make $\Gamma(M)$ into a bialgebra.

2.6. Decompositions. The symmetric algebra $S(M)$ is defined as the free commutative $\mathbb{k}$-algebra generated by $M$ and has a decomposition

$$
S(M)=\bigoplus_{d \in \mathbb{N}_{0}} \operatorname{Sym}_{d} M
$$

It follows that $S(-)$ defines a functor from $\mathcal{P}_{\mathbb{k}}$ to the category of all commutative $\mathbb{k}$-algebras, which preserves coproducts. Hence $S(M) \otimes S(N) \cong S(M \oplus N)$, and by the duality (2.4) there is an isomorphism

$$
\Gamma(M) \otimes \Gamma(N) \simeq \Gamma(M \oplus N) .
$$

The isomorphism (2.6) is given explicitly by restricting the multiplication map $x \otimes y \mapsto x * y$, where $\Gamma(M), \Gamma(N)$ are considered as subalgebras of $\Gamma(M \oplus N)$. It follows that for each $d \in \mathbb{N}_{0}$ there is a decomposition

$$
\Gamma^{d}(M \oplus N)=\bigoplus_{0 \leq c \leq d} \Gamma^{c}(M) * \Gamma^{d-c}(N)
$$

where $\Gamma^{c}(M) * \Gamma^{d-c}(N)$ denotes the image of $\Gamma^{c}(M) \otimes \Gamma^{d-c}(N)$ under (2.6).

Note that $\Gamma^{d} \mathbb{k} \cong \mathbb{k}$ for all $d \in \mathbb{N}_{0}$. Thus, given a free $\mathbb{k}$-module $V$ of finite rank, it follows by induction from (2.7) that the divided power $\Gamma^{d} V$ is again a free $\mathbb{k}$-module of finite rank. For example, suppose $V$ has a finite ordered $\mathbb{k}$-basis $\left\{x_{b}\right\}_{b \in \mathcal{B}}$. Then $\Gamma^{d} V$ has the following $\mathbb{k}$-basis

$$
\left\{x^{\mu}:=\prod_{b \in \mathcal{B}} x_{b}^{\otimes \mu_{b}} \mid \mu \in \Lambda_{d}(\mathcal{B})\right\}
$$

where the product denotes multiplication in $\Gamma(V)$.

The basis $(2.8)$ can also be parameterized by elements of $\operatorname{seq}^{d}(\mathcal{B})$. First notice that the the tensor power $V^{\otimes d}$ has the following basis

$$
\left\{x_{\otimes \mathrm{b}}:=x_{b_{1}} \otimes \ldots \otimes x_{b_{d}} \mid \mathrm{b} \in \operatorname{seq}^{d}(\mathcal{B})\right\} .
$$

Given $\mathrm{b} \in \operatorname{seq}^{d}(\mathcal{B})$, we then define $x_{\mathrm{b}}:=\sum_{\mathrm{b} \sim \mathrm{c}} x_{\otimes \mathrm{c}}$. Notice that $x_{\mathrm{b}}=x_{\mu(\mathrm{b})}$. It then follows from Lemma 2.3 that the set

$$
\left\{x_{\mathrm{b}} \mid \mathrm{b} \in \operatorname{seq}^{d}(\mathcal{B}) / \mathfrak{S}_{d}\right\}
$$

is also a basis of $\Gamma^{d} V$, indexed by any complete set of orbit representatives. 
2.7. Polynomial functors. We recall the definitions of some well known polynomial endofunctors on the category $\mathcal{P}_{\mathbb{k}}$ along with their associated natural transformations.

Let $d \in \mathbb{N}_{0}$. Then recall the functor $\otimes^{d}: \mathcal{P}_{\mathbb{k}} \rightarrow \mathcal{P}_{\mathbb{k}}$ sending $M \mapsto M^{\otimes d}$, whose action on morphisms is defined by

$$
\otimes_{M, N}^{d}(\varphi):=\varphi \otimes \cdots \otimes \varphi: M^{\otimes d} \rightarrow N^{\otimes d}
$$

for any $\varphi \in \operatorname{Hom}(M, N)$.

It follows easily from (2.7) that the divided power $\Gamma^{d} M$ of a finitely-generated, projective $\mathbb{k}$-module $M \in \mathcal{P}_{\mathbb{k}}$ is again finitely-generated and projective. This yields a functor $\Gamma^{d}: \mathcal{P}_{\mathbb{k}} \rightarrow \mathcal{P}_{\mathbb{k}}$ which is a subfunctor of $\otimes^{d}$. In particular, the action of $\Gamma^{d}$ on morphisms is defined by restriction

$$
\Gamma_{M, N}^{d}(\varphi):=\left.\left(\varphi^{\otimes d}\right)\right|_{\Gamma^{d} M}: \Gamma^{d} M \rightarrow \Gamma^{d} N
$$

for any $\varphi \in \operatorname{Hom}(M, N)$.

Now let $S, T: \mathcal{P}_{\mathbb{k}} \rightarrow \mathcal{P}_{\mathbb{k}}$ be an arbitrary pair of functors. Then the tensor product $-\otimes-$ induces the following bifunctors

$$
S \otimes T, \quad T(-\otimes-): \mathcal{P}_{\mathbb{k}} \times \mathcal{P}_{\mathbb{k}} \rightarrow \mathcal{P}_{\mathbb{k}}
$$

which are respectively defined by

$$
S \otimes T:=(-\otimes-) \circ(S \times T), \quad T(-\otimes-):=T \circ(-\otimes-) .
$$

We also have the "object-wise" tensor product $S \otimes T: \mathcal{P}_{\mathbb{k}} \rightarrow \mathcal{P}_{\mathbb{k}}$ defined by

$$
S \otimes T:=(S \otimes T) \circ \delta
$$

where $\delta: \mathcal{P}_{\mathbb{k}} \rightarrow \mathcal{P}_{\mathbb{k}} \times \mathcal{P}_{\mathbb{k}}$ denotes the diagonal embedding: $M \mapsto(M, M)$.

Now suppose $M, N \in \mathcal{P}_{\mathbb{k}}$. As in [17], define $\psi^{d}=\psi^{d}(M, N)$ to be the unique map which makes the following square commute:

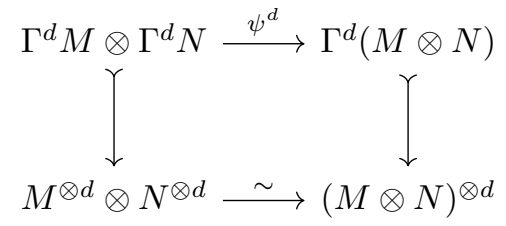

The following lemma is easy to check.

Lemma 2.5. (1) The maps $\psi^{d}(M, N)$ form a natural transformation of bifunctors

$$
\psi^{d}: \Gamma^{d} \otimes \Gamma^{d} \rightarrow \Gamma^{d}(-\otimes-) .
$$

(2) If $M, N \in \mathcal{P}_{\mathbb{k}}$, then the following diagram commutes

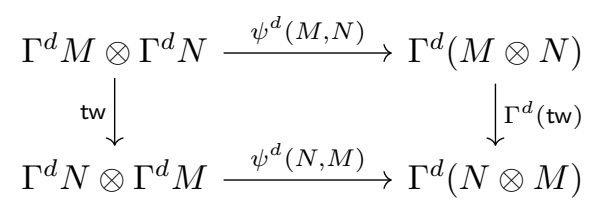

where tw permutes tensor factors as in (2.5).

\section{Generalized Schur Algebras}

After recalling the definition of generalized Schur algebras [4] associated to a $\mathbb{k}$ algebra $A$, we introduce corresponding standard homomorphisms between certain modules of divided powers. 
3.1. Associative $\mathbb{k}$-algebras. Suppose that $R, S$ are associative algebras in the category $\mathcal{M}_{\mathbb{k}}$. Recall that the tensor product $R \otimes S$ is the algebra in $\mathcal{M}_{\mathbb{k}}$ with multiplication $m_{R \otimes S}$ defined by

$$
R \otimes S \otimes R \otimes S \stackrel{1 \otimes \mathrm{tw} \otimes 1}{\longrightarrow} R \otimes R \otimes S \otimes S \stackrel{m_{R} \otimes m_{S}}{\longrightarrow} R \otimes S .
$$

Given $d \in \mathbb{N}$, the tensor power $R^{\otimes d}$ is an associative algebra in $\mathcal{M}_{\mathbb{k}}$ in a similar way. If $R$ is unital, then $R^{\otimes d}$ has unit $1_{R}^{\otimes d}$.

In the remainder, the term $\mathbb{k}$-algebra will always refer to a unital, associative algebra in the category $\mathcal{P}_{\mathbb{k}}$. Let $A \in \mathcal{P}_{\mathbb{k}}$ be a $\mathbb{k}$-algebra. Then $A$-mod (resp. mod- $A$ ) denotes the subcategory of $\mathcal{P}_{\mathbb{k}}$ consisting of all left (right) $A$-modules, $M \in \mathcal{P}_{\mathbb{k}}$, and $A$-module homomorphisms. Write $\operatorname{Hom}_{A}(M, N) \in \mathcal{P}_{\mathbb{k}}$ to denote the set of all $A$-homomorphisms from $M$ to $N$ for $M, N \in A$-mod (resp. $\bmod -A$ ). We also write $\rho_{M}: A \otimes M \rightarrow A$ (resp. $\rho_{M}: M \otimes A \rightarrow A$ ) to denote the induced linear map corresponding to a left (right) $A$-module.

If $M \in A$-mod (resp. $\bmod -A)$ and $N \in B$-mod (resp. mod- $B$ ), the tensor product $M \otimes N$ is a left (resp. right) $A \otimes B$-module, with corresponding module map: $\rho_{M \otimes N}=\left(\rho_{M} \otimes \rho_{N}\right) \circ(1 \otimes \tau \otimes 1)$.

3.2. The algebra $\Gamma^{d} A$. Suppose $A$ is a $\mathbb{k}$-algebra. Then $\Gamma^{d} A$ is a $\mathbb{k}$-algebra with multiplication $m_{\Gamma^{d} A}$ defined via the composition

$$
\Gamma^{d} A \otimes \Gamma^{d} A \stackrel{\psi^{d}}{\longrightarrow} \Gamma^{d}(A \otimes A) \stackrel{\Gamma^{d}\left(m_{A}\right)}{\longrightarrow} \Gamma^{d} A
$$

where the second map denotes the functorial action of $\Gamma^{d}$ on $m_{A}$. It follows that $\Gamma^{d} A$ is a unital subalgebra of $A^{\otimes d}$.

Example 3.1 (The Schur algebra). Suppose $n \in \mathbb{N}$, and let $\mathrm{M}_{n}(\mathbb{k})$ denote the algebra of all $n \times n$-matrices in $\mathbb{k}$. Then $\Gamma^{d} \mathrm{M}_{n}(\mathbb{k})$ is isomorphic to the classical $S c h u r$ algebra, $S(n, d)$, defined by Green [9, Theorem 2.6c]. We view this isomorphism as an identification.

We now have two distinct multiplications on the direct sum $\Gamma(A)=\bigoplus_{d \in \mathbb{N}} \Gamma^{d} A$. In order to distinguish them, we sometimes refer to the shuffle product

$$
\nabla: \Gamma^{d} A \otimes \Gamma^{e} A \rightarrow \Gamma^{d+e} A: x \otimes y \mapsto x * y
$$

as outer multiplication in $\Gamma(A)$, while inner multiplication refers to the map defined as multiplication in $\Gamma^{d} A$ on diagonal components

$$
m_{\Gamma^{d} A}: \Gamma^{d} A \otimes \Gamma^{d} A \rightarrow \Gamma^{d} A: x \otimes y \mapsto x y
$$

and then extended by zero to other components.

3.3. Generalized Schur algebras. Given a $\mathbb{k}$-algebra $A$, write $\mathrm{M}_{n}(A)$ for the algebra of $n \times n$-matrices in $A$. We identify $\mathrm{M}_{n}(A)$ with $\mathrm{M}_{n}(\mathbb{k}) \otimes A$ via

$$
\mathrm{M}_{n}(A) \stackrel{\sim}{\longrightarrow} \mathrm{M}_{n}(\mathbb{k}) \otimes A:\left(a_{i j}\right) \mapsto \sum_{i, j} E_{i j} \otimes a_{i j},
$$

where $E_{i j}$ are elementary matrices in $\mathrm{M}_{n}(\mathbb{k})$. Next, suppose $V$ is any left (resp. right) $\mathrm{M}_{n}(\mathbb{k})$-module, and let $M \in A$-mod $(\bmod -A)$. Then write $V(M):=V \otimes M$ to denote the corresponding $\mathrm{M}_{n}(A)$-module.

Definition 3.2. Suppose $A$ is an algebra, and let $n \in \mathbb{N}, d \in \mathbb{N}_{0}$. Then the generalized Schur algebra $S^{A}(n, d)$ is the algebra $\Gamma^{d} \mathrm{M}_{n}(A)$.

Using the notation of [4], notice that $\mathrm{M}_{n}$ is spanned by the elements $\xi_{i, j}^{a}:=$ $E_{i j} \otimes a$, for all $a \in A$ and $i, j \in[1, n]$. Now suppose that $A$ is free as a $\mathbb{k}$-module with finite ordered basis $\left\{x_{b}\right\}_{b \in \mathcal{B}}$. Then $\mathrm{M}_{n}(A)$ has a corresponding basis

$$
\left\{\xi_{i, j, b}:=\xi_{i, j}^{x_{b}} \mid i, j \in[1, n], b \in \mathcal{B}\right\} .
$$


We view $\mathrm{M}_{n}(\mathbb{k})$ as a subalgebra of $\mathrm{M}_{n}(A)$ by identifying $E_{i j}=\xi_{i, j}^{1}$. Notice that the classical Schur algebra $S(n, d)$ is thus a (unital) subalgebra of $S^{A}(n, d)$.

For each triple $(i, j, b) \in \operatorname{seq}^{d}(n, n, \mathcal{B})$, there is a corresponding element of $S^{A}(n, d)$ denoted by

$$
\xi_{\mathrm{i}, \mathrm{j}, \mathrm{b}}:=\sum_{(\mathrm{i}, \mathrm{j}, \mathrm{b}) \sim(\mathrm{r}, \mathrm{s}, \mathrm{c})} \xi_{r_{1}, s_{1}, c_{1}} \otimes \cdots \otimes \xi_{r_{d}, s_{d}, c_{d}},
$$

where the sum is over all triples $(r, s, c)$ in the same diagonal $\mathfrak{S}_{d^{-}}$orbit as $(i, j, b)$. It thus follows from (2.2), (2.8) and (2.9) that the set

$$
\left\{\xi_{i, j, b} \mid(i, j, b) \in \operatorname{seq}^{d}(n, n, \mathcal{B}) / \mathfrak{S}_{d}\right\}
$$

forms a basis of of $S^{A}(n, d)$. In a similar way, the subalgebra $S(n, d)$ has a basis given by

$$
\left\{\xi_{\mathrm{i}, \mathrm{j}}:=\sum_{(\mathrm{i}, \mathrm{j}) \sim(\mathrm{r}, \mathrm{s})} \xi_{r_{1}, s_{1}}^{1} \otimes \cdots \otimes \xi_{r_{d}, s_{d}}^{1} \mid(\mathrm{i}, \mathrm{j}) \in \operatorname{seq}^{d}(n, n) / \mathfrak{S}_{d}\right\} .
$$

For each weight $\mu \in \Lambda_{d}(n)$, we write

$$
\xi_{\mu}:=\xi_{i_{\mu}, \dot{i}_{\mu}}
$$

to denote the corresponding idempotent in $S(n, d) \subset S^{A}(n, d)$.

3.4. Standard homomorphisms. Let us fix an algebra $A$ throughout the remainder of the section. Given $M \in A$-mod, it follows from (2.11) that $\Gamma^{d} M$ is a left $\Gamma^{d} A$-module with module map $\rho_{\Gamma^{d} M}$ determined by the composition

$$
\Gamma^{d} A \otimes \Gamma^{d} M \stackrel{\psi^{d}}{\longrightarrow} \Gamma^{d}(A \otimes M) \stackrel{\Gamma^{d}\left(\rho_{M}\right)}{\longrightarrow} \Gamma^{d}(M),
$$

where the second map denotes the functorial action of $\Gamma^{d}$ on $\rho_{M}$.

Lemma 3.3. Suppose $M, N \in A$-mod, and let $\varphi: M \rightarrow N$ be an A-module homomorphism. Then the functorial map

$$
\Gamma^{d}(\varphi): \Gamma^{d} M \rightarrow \Gamma^{d} N
$$

is a homomorphism of $\Gamma^{d} A$-modules. Moreover, if $\varphi$ is injective (resp. surjective) then so is $\Gamma^{d}(\varphi)$.

Proof. The map $\varphi^{\otimes d}: M^{\otimes d} \rightarrow N^{\otimes d}$ is a homomorphism of $A^{\otimes d}$-modules, and if $\varphi$ is injective (resp. surjective) then so is $\varphi^{\otimes d}$. The statements for $\Gamma^{d}(\varphi)$ follow by restriction.

Suppose $d, e \in \mathbb{N}_{0}$ and $M, N \in A$-mod. Notice that the homogeneous component of comultiplication

$$
\Delta: \Gamma^{d+e} A \rightarrow \Gamma^{d} A \otimes \Gamma^{e} A
$$

is an injective (unital) map of $\mathbb{k}$-algebras. It follows that $\Gamma^{d} M \otimes \Gamma^{e} N$ has a corresponding $\Gamma^{d} A$-module structure, defined by restriction along (3.1). In the particular case $M=N$, we note that each of the following maps is a $\Gamma^{d} A$-module homomorphism:

$$
\begin{gathered}
\Delta: \Gamma^{d+e} M \rightarrow \Gamma^{d} M \otimes \Gamma^{e} M, \quad \nabla: \Gamma^{d} M \otimes \Gamma^{e} M \rightarrow \Gamma^{d+e} M, \\
\text { tw }: \Gamma^{d} M \otimes \Gamma^{e} M \stackrel{\sim}{\rightarrow} \Gamma^{e} M \otimes \Gamma^{d} M,
\end{gathered}
$$

where $\nabla$ (resp. $\Delta$ ) are components of (co)multiplication in the bialgebra $\Gamma(M)$. Setting $A=\mathbb{k}$ then gives the following. 
Lemma 3.4. Let $d, e \in \mathbb{N}$. Then there are natural transformations

$$
\Delta: \Gamma^{d+e} \rightarrow \Gamma^{d} \otimes \Gamma^{e}, \quad \nabla: \Gamma^{d} \otimes \Gamma^{e} \rightarrow \Gamma^{d+e}
$$

of functors $\mathcal{P}_{\mathbb{k}} \rightarrow \mathcal{P}_{\mathbb{k}}$ induced by setting $\Delta(M)$ (resp. $\nabla(M)$ ) equal to (co)multiplication in $\Gamma(M)$, for each $M \in \mathcal{P}_{\mathbb{k}}$.

Now suppose $r \in \mathbb{N}$ and $\mu \in \Lambda(r)$. Given $M, N_{1}, \ldots, N_{r} \in \mathcal{P}_{\mathbb{k}}$, we write

$$
\Gamma^{(\mu)}\left(N_{1}, \ldots, N_{r}\right):=\Gamma^{\mu_{1}} N_{1} \otimes \cdots \otimes \Gamma^{\mu_{r}} N_{r}
$$

and set

$$
\Gamma^{\mu} M:=\Gamma^{(\mu)}(M, \ldots, M) .
$$

If $M_{1}, \ldots, M_{r} \in A$-mod, then we consider $\Gamma^{(\mu)}\left(M_{1}, \ldots, M_{r}\right)$ as a left $\Gamma^{d} A$-module by restriction along the corresponding inclusion, $\Delta: \Gamma^{d} A \rightarrow \Gamma^{\mu} A$, of $\mathbb{k}$-algebras.

Suppose that $\gamma=\left(\gamma_{i j}\right) \in \Lambda_{d}(\mathbb{N} \times \mathbb{N})$ is a (semi-infinite) matrix whose entries sum to $d$. Then let $\lambda, \mu \in \Lambda_{d}(\mathbb{N})$ be weights such that $\lambda_{i}=\sum_{j} \gamma_{i j}$ and $\mu_{j}=\sum_{i} \gamma_{i j}$ for all $i, j \in \mathbb{N}$. Slightly abusing notation, for a given $N \in \mathcal{P}_{\mathbb{k}}$, we also write $\gamma=\gamma(N)$ to denote the corresponding standard homomorphism:

$$
\gamma: \Gamma^{\mu} N \rightarrow \Gamma^{\lambda} N
$$

defined by the composition

$$
\bigotimes_{j} \Gamma^{\mu_{j}} N \stackrel{\Delta \otimes \ldots \otimes \Delta}{\longrightarrow} \bigotimes_{i} \bigotimes_{j} \Gamma^{\gamma_{i j}} N \stackrel{\sim}{\longrightarrow} \bigotimes_{j} \bigotimes_{i} \Gamma^{\gamma_{i j}} N \stackrel{\nabla \otimes \ldots \otimes \nabla}{\longrightarrow} \bigotimes_{i} \Gamma^{\lambda_{i}} N,
$$

where each $\nabla$ (resp. $\Delta$ ) denotes an appropriate component of (co)multiplication in the bialgebra $\Gamma(N)$, and where the second map rearranges the tensor factors.

If $M \in A$-mod, then it follows from (3.2) that $\gamma(M): \Gamma^{\mu} M \rightarrow \Gamma^{\lambda} M$ is a homomorphism of $\Gamma^{d} A$-modules. In the same way, we obtain homomorphisms of $S^{A}(n, d)$-modules corresponding to any given $M \in \mathrm{M}_{n}(A)$-mod.

3.5. Quotient modules. Suppose $M \in \mathcal{P}_{\mathbb{k}}$. Then we write $\langle L\rangle \subset M^{\otimes d}$ to denote the $\mathfrak{S}_{d}$-submodule generated by a subset $L \subset M^{\otimes d}$. For example if $L_{1}, \ldots, L_{d} \subset M$ are $\mathbb{k}$-submodules and $L=L_{1} \otimes \cdots \otimes L_{d}$, then

$$
\langle L\rangle=\sum_{\sigma \in \mathfrak{S}_{d}} L_{1 \sigma} \otimes \cdots \otimes L_{d \sigma}
$$

where $i \sigma:=\sigma^{-1}(i)$ denotes the right action of $\sigma$ on $i \in[1, d]$.

Now suppose $M=N \oplus N^{\prime}$ for some $\mathbb{k}$-submodules $N, N^{\prime} \subset N$. Then notice that there is a corresponding decomposition

$$
M^{\otimes d}=\left(N^{\prime}\right)^{\otimes d} \oplus\left\langle N \otimes M^{\otimes d-1}\right\rangle,
$$

which is a direct sum of $\mathfrak{S}_{d}$-submodules. Taking $\mathfrak{S}_{d}$-invariants on both sides results in the decomposition

$$
\Gamma^{d} M=\Gamma^{d}\left(N^{\prime}\right) \oplus\left\langle N \otimes M^{\otimes d-1}\right\rangle^{\mathfrak{S}_{d}}
$$

into $\mathbb{k}$-submodules. The decomposition (3.3) then makes it possible to describe the kernel of the quotient map

$$
\Gamma^{d}(\pi): \Gamma^{d} M \rightarrow \Gamma^{d}(M / N)
$$

induced by projection $\pi: M \rightarrow M / N$. More generally, we note the following. 
Lemma 3.5. Let $A$ be a $\mathbb{k}$-algebra. Suppose $N \subset M$ is an inclusion of $A$-modules such that $M=N \oplus N^{\prime}$ for some $\mathbb{k}$-submodule $N^{\prime} \subset M$. Then there is an exact sequence

$$
0 \rightarrow\left\langle N \otimes M^{\otimes d-1}\right\rangle^{\mathfrak{S}_{d}} \longrightarrow \Gamma^{d} M \stackrel{\Gamma^{d}(\pi)}{\longrightarrow} \Gamma^{d}(M / N) \rightarrow 0
$$

of $\Gamma^{d} A$-module homomorphisms.

Proof. It follows from (3.3) that the required exact sequence of $\Gamma^{d} A$-modules is obtained by restriction from the exact sequence

$$
0 \rightarrow\left\langle N \otimes M^{\otimes d-1}\right\rangle \longrightarrow M^{\otimes d} \stackrel{\pi^{\otimes d}}{\longrightarrow}(M / N)^{\otimes d} \rightarrow 0
$$

of $A^{\otimes d}$-module homomorphisms.

We introduce some additional notation. Suppose $N_{1}, \ldots, N_{r} \subset M$ is a finite collection of $\mathbb{k}$-submodules of some $M \in \mathcal{P}_{\mathbb{k}}$, and let $\mu \in \Lambda_{r}(d)$. Then we write

$$
N_{\otimes \mu}:=N_{1}^{\otimes \mu_{1}} \otimes \ldots \otimes N_{r}^{\otimes \mu_{r}}
$$

to denote the corresponding $\mathbb{k}$-submodule of $M^{\otimes d}$ and use the notation

$$
N_{\mu}:=\left\langle N_{\otimes \mu}\right\rangle^{\mathfrak{S}_{d}} \subset \Gamma^{d} M
$$

for the $\mathbb{k}$-submodule of $\mathfrak{S}_{d}$-invariants.

\section{Wreath Products and Generalized Schur-Weyl Duality}

Let us briefly recall the generalized Schur-Weyl duality [4] which establishes a relationship between a wreath product algebra $A \succ \mathfrak{S}_{d}$ and a corresponding $A$-Schur algebra via their respective actions on a common tensor space.

4.1. Wreath products. Fix a $\mathbb{k}$-algebra $A$. The wreath product algebra $A$ ? $\mathfrak{S}_{d}$ is the $\mathbb{k}$-module $A^{\otimes d} \otimes \mathbb{k} \mathfrak{S}_{d}$, with multiplication defined by

$$
(x \otimes \rho) \cdot(y \otimes \sigma):=x\left(y \rho^{-1}\right) \otimes \rho \sigma
$$

for all $x, y \in A^{\otimes d}$ and $\rho, \sigma \in \mathfrak{S}_{d}$. If $G$ is a finite group, then note for example that $(\mathbb{k} G)\left\{\mathfrak{S}_{d}\right.$ is isomorphic to the group algebra of the classical wreath product, $G$ < $\mathfrak{S}_{d}:=G^{d} \rtimes \mathfrak{S}_{d}$.

Assume for the rest of the section that $A$ is free as a $\mathbb{k}$-module. We then identify the tensor power $A^{\otimes d}$ and group algebra $\mathbb{k} \mathfrak{S}_{d}$ as subalgebras of $A$ < $\mathfrak{S}_{d}$ by setting

$$
A^{\otimes d}=A^{\otimes d} \otimes 1_{\mathfrak{S}_{d}}, \quad \mathbb{k} \mathfrak{S}_{d}=1_{A} \otimes d \otimes \mathbb{k} \mathfrak{S}_{d}
$$

respectively.

4.2. Generalized Schur-Weyl duality. Suppose $n, d \in \mathbb{N}$. Write $\mathrm{V}_{n}:=\mathbb{k}^{n}$ to denote the standard left $\mathrm{M}_{n}(\mathbb{k})$-module, with basis elements

$$
v_{i}:=(0, \ldots, 1, \ldots, 0)
$$

for $i \in[1, n]$, considered as column vectors. Then for simplicity, let us write

$$
\mathrm{V}:=\mathrm{V}_{n}(A)=\mathbb{k}^{n} \otimes A
$$

to denote the corresponding left $\mathrm{M}_{n}(A)$-module.

We may identify $\mathrm{V}$ and $A^{n}$ as right $A$-modules, and it follows that the tensor space, $\mathrm{V}^{\otimes d}$, is naturally a right $A^{\otimes d}$-module. A right action of $A \succ \mathfrak{S}_{d}$ on $\mathrm{V}^{\otimes d}$ is then defined by setting

$$
w(x \cdot \sigma):=(w x) \sigma, \quad \text { for } w \in \mathrm{V}^{\otimes d}, x \in A^{\otimes d} \text {, and } \sigma \in \mathfrak{S}_{d} .
$$


More explicitly, suppose $w=w_{1} \otimes \cdots \otimes w_{d}$ and $x=x_{1} \otimes \cdots \otimes x_{d}$, for some $w_{i} \in \mathrm{V}$ and $x_{i} \in A$. Then notice that

$$
(w x) \sigma=\left(w_{1 \sigma} x_{1 \sigma}\right) \otimes \cdots \otimes\left(w_{d \sigma} x_{d \sigma}\right)=(w \sigma)(x \sigma)
$$

for any $\sigma \in \mathfrak{S}_{d}$. Hence, by (4.1) we have

$$
w(\sigma \cdot x)=w\left(\left(x \sigma^{-1}\right) \cdot \sigma\right)=\left(w\left(x \sigma^{-1}\right)\right) \sigma=(w \sigma) x .
$$

It follows that (4.2) is well-defined.

Lemma 4.1 ([4, Lemma 5.7]). The embedding $S^{A}(n, d) \hookrightarrow \mathrm{M}_{n}(A)^{\otimes d} \cong \operatorname{End}_{A^{\otimes d}}\left(\mathrm{~V}^{\otimes d}\right)$ defines an algebra isomorphism

$$
S^{A}(n, d) \cong \operatorname{End}_{A l \mathfrak{S}_{d}}\left(\mathrm{~V}^{\otimes d}\right)
$$

for all $n, d \in \mathbb{N}$.

Given $n \geq d$, let $\omega \in \Lambda_{d}(n)$ denote the weight $\omega=\left(1^{d}\right)=(1, \ldots, 1,0, \ldots, 0)$. Then considering $\mathrm{V}$ again as a left $\mathrm{M}_{n}(A)$-module, notice that $\mathrm{V}^{\otimes d}$ is equal to the left $S^{A}(n, d)$-module $\Gamma^{\omega} \mathrm{V}$.

For each weight $\mu \in \Lambda_{d}(n)$, define a corresponding element

$$
v_{\otimes \mu}:=v_{1}^{\otimes \mu_{1}} \otimes \ldots \otimes v_{n}^{\otimes \mu_{n}}
$$

in the tensor space $\mathrm{V}^{\otimes d}$.

The next result summarizes (5.15) and (5.17) of [4].

Proposition $4.2([4])$. Assume that $n \geq d$.

(i) There is a unique $\left(S^{A}(n, d), A<\mathfrak{S}_{d}\right)$-bimodule isomorphism $S^{A}(n, d) \xi_{\omega} \stackrel{\sim}{\rightarrow}$ $\mathrm{V}^{\otimes d}$ which maps $\xi_{\omega} \mapsto v_{\otimes \omega}$.

(ii) There is an algebra isomorphism, $A \nmid \mathfrak{S}_{d} \stackrel{\sim}{\rightarrow} \xi_{\omega} S^{A}(n, d) \xi_{\omega}$, given by:

$$
\left(x_{1} \otimes \ldots \otimes x_{d}\right) \otimes \sigma \mapsto \xi_{1,1 \sigma}^{x_{1}} * \cdots * \xi_{d, d \sigma}^{x_{d}} .
$$

(iii) $\operatorname{End}_{S^{A}(n, d)}\left(\mathrm{V}^{\otimes d}\right) \cong A$ 乙 $\mathfrak{S}_{d}$.

\section{Cauchy Decompositions}

The Cauchy decomposition for symmetric algebras via Schur modules [1] is an analogue of Cauchy's formula for symmetric functions $[2,18]$. A corresponding decomposition for divided powers $[12,17]$ is defined in terms of Weyl (or co-Schur) modules. In this section, we describe a generalized Cauchy decomposition (Theorem 5.14 ) for divided powers of an $(A, B)$-bimodule with respect to a given filtration on the bimodule.

5.1. Weyl modules. Weyl modules are defined in [1, Definition II.1.4] as the image of a single map from a tensor product of divided powers of a module into a tensor product of exterior powers. We use an equivalent definition from the proof of $[1$, Theorem II.3.16]) which involves quotients of divided powers.

Throughout the section, we fix some $d \in \mathbb{N}$. Suppose $\lambda \in \Lambda_{d}(\mathbb{N})$, and let $M \in \mathcal{P}_{\mathbb{k}}$. For each pair $(i, t)$ with $1 \leq i<l(\lambda)$ and $1 \leq t \leq \lambda_{i+1}$, let us write

$$
\lambda(i, t)=\left(\lambda_{1}, \ldots, \lambda_{i-1}, \lambda_{i}+t, \lambda_{i+1}-t, \lambda_{i+1}, \ldots, \lambda_{m}\right) \in \Lambda_{d}(\mathbb{N}) .
$$

Then write $\gamma_{\lambda(i, t)}: \Gamma^{\lambda(i, t)} M \rightarrow \Gamma^{\lambda} M$ to denote the standard homomorphism corresponding to the matrix

$$
\gamma_{\lambda(i, t)}:=\operatorname{diag}\left(\lambda_{1}, \lambda_{2}, \ldots\right)+t E_{i+1, i}-t E_{i+1, i} .
$$

Similarly, let $\gamma_{\lambda(i, t)}^{\mathrm{tr}}: \Gamma^{\lambda} M \rightarrow \Gamma^{\lambda(i, t)} M$ denote the map corresponding to the transpose of the above matrix. 
Definition 5.1 ([1]). Suppose $M \in \mathcal{P}_{\mathbb{k}}$ and $\lambda \in \Lambda_{d}^{+}(\mathbb{N})$. Let $\square_{\lambda}(M)$ denote the $\mathbb{k}$-submodule of $\Gamma^{\lambda} M$ defined by

$$
\square_{\lambda}(M):=\sum_{i \geq 1} \sum_{t=1}^{\lambda_{t+1}} \operatorname{Im}\left(\gamma_{\lambda(i, t)}\right) \subset \Gamma^{\lambda} M .
$$

The Weyl module, $W_{\lambda}(M)$, is defined as the quotient $\mathbb{k}$-module

$$
W_{\lambda}(M):=\Gamma^{\lambda} M / \square_{\lambda}(M) .
$$

Let $A$ be a $\mathbb{k}$-algebra and suppose now that $M \in A$-mod. Then $\square_{\lambda}(M)$ is a $\Gamma^{d} A$ submodule of $\Gamma^{\lambda} M$, since the standard homomorphisms are $\Gamma^{d} A$-module maps. It follows that $W_{\lambda}(M)$ is a $\Gamma^{d} A$-module. In particular, $W_{\lambda}\left(\mathbb{k}^{n}\right)$ is an $S(n, d)$-module.

5.2. The standard basis. Consider a fixed partition $\lambda=\left(\lambda_{1}, \lambda_{2}, \ldots\right) \in \Lambda_{d}^{+}(\mathbb{N})$. The Young diagram of $\lambda$ is the following subset of $\mathbb{N} \times \mathbb{N}$ :

$$
[\lambda]:=\left\{(i, j) \mid 1 \leq i \leq l(\lambda), 1 \leq j \leq \lambda_{i}\right\} .
$$

Suppose $\mathcal{B}$ is a finite totally ordered set. Let $\operatorname{Tab}_{\lambda}(\mathcal{B})$ denote the set of all functions $\mathrm{T}:[\lambda] \rightarrow \mathcal{B}$, called tableaux (of shape $\lambda$ ).

A tableau $\mathrm{T}$ will be identified with the diagram obtained by placing each value $\mathrm{T}_{i, j}:=\mathrm{T}(i, j)$ in the $(i, j)$-th entry of $[\lambda]$. For example if $\mathrm{T} \in \operatorname{Tab}_{(3,2)}(\mathcal{B})$, then we write

$$
\mathrm{T}=\begin{array}{lll}
\mathrm{T}_{1,1} & \mathrm{~T}_{1,2} & \mathrm{~T}_{1,3} \\
\mathrm{~T}_{2,1} & \mathrm{~T}_{2,2}
\end{array}
$$

We say that a tableau $\mathrm{T}$ is row (column) standard if each row (column) is a nondecreasing (increasing) function of $i$ (resp. $j$ ), and $\mathrm{T}$ is standard if it is both row and column standard.

Let $\operatorname{St}_{\lambda}(\mathcal{B}) \subset \operatorname{Tab}_{\lambda}(\mathcal{B})$ denote the subset of all standard tableaux. This subset is nonempty if and only if $l(\lambda) \leq \sharp \mathcal{B}$. In particular, suppose $l(\lambda) \leq \sharp \mathcal{B}$ and assume the elements of $\mathcal{B}$ are listed as in (2.1). Then we write $\mathrm{T}^{\lambda}=\mathrm{T}^{\lambda}(\mathcal{B})$ to denote the standard tableau in $\operatorname{St}_{\lambda}(\mathcal{B})$ with entries $\mathrm{T}_{i, j}^{\lambda}:=b_{i}^{\mathcal{B}}$ for all $(i, j) \in[\lambda]$. For example, if $d=7, \lambda=(4,2,1)$ and $\mathcal{B}=[1,3]$, then

$$
\mathrm{T}^{\lambda}=\begin{array}{llll}
1 & 1 & 1 & 1 \\
2 & 2 & & \\
3 & &
\end{array}
$$

Fix a free $\mathbb{k}$-module $V$ with finite ordered basis $\left\{x_{b}\right\}_{b \in \mathcal{B}}$. If $\mathrm{T} \in \operatorname{Tab}_{\lambda}(\mathcal{B})$, then for $q=l(\lambda)$ and $i \in[1, q]$ we write

$$
\mathrm{T}_{i}:=\mathrm{T}(i,-) \in \operatorname{seq}^{\lambda_{i}}(\mathcal{B})
$$

to denote the to the $i$-th row of $\mathrm{T}$, and we set

$$
x_{\mathrm{T}}:=x_{\mathrm{T}_{1}} \otimes \cdots \otimes x_{\mathrm{T}_{q}} \in \Gamma^{\lambda} V .
$$

Notice that the set of $x_{\mathrm{T}}$ paramaterized by all row standard $\mathrm{T} \in \mathrm{Tab}_{\lambda}(\mathcal{B})$ forms a basis of $\Gamma^{\lambda} V$.

The following result describes a basis for Weyl modules.

Proposition 5.2 ([1], Theorem III.3.16). Let $\lambda \in \Lambda^{+}(\mathbb{N})$ and suppose $V$ is a free $\mathbb{k}$-module with a finite ordered basis $\left\{x_{b}\right\}_{b \in \mathcal{B}}$. Then the Weyl module $W_{\lambda}(V)$ is also a free $\mathbb{k}$-module, with basis given by the set of images

$$
\left\{\bar{x}_{\mathrm{T}}:=\pi\left(x_{\mathrm{T}}\right) \mid \mathrm{T} \in \mathrm{St}_{\lambda}(\mathcal{B})\right\}
$$

under the canonical projection $\pi: \Gamma^{\lambda} V \rightarrow \Gamma^{\lambda} V / \square_{\lambda}(V)$. 
This result shows for example that the Weyl module $W_{\lambda}(V)$ is nonzero if and only if $l(\lambda) \leq \sharp \mathcal{B}$. Another consequence of the proposition is that $W_{\lambda}(M)$ is a projective $\mathbb{k}$-module for any $M \in \mathcal{P}_{\mathbb{k}}$ (cf. [17, p. 1013]).

5.3. The Cauchy decomposition. Suppose $M, N \in \mathcal{P}_{\mathbb{k}}$. The maps $\psi^{d}$ appearing in (2.11) can be generalized as follows. If $\lambda \in \Lambda_{d}^{+}(\mathbb{N})$, let

$$
\psi^{\lambda}(M, N): \Gamma^{\lambda} M \otimes \Gamma^{\lambda} N \rightarrow \Gamma^{d}(M \otimes N)
$$

denote the map defined via the composition

$$
\begin{aligned}
\Gamma^{\lambda} M \otimes \Gamma^{\lambda} N & \stackrel{\sim}{\longrightarrow}\left(\Gamma^{\lambda_{1}} M \otimes \Gamma^{\lambda_{1}} N\right) \otimes \ldots \otimes\left(\Gamma^{\lambda_{m}} M \otimes \Gamma^{\lambda_{m}} N\right) \\
& \stackrel{\psi \otimes \ldots \otimes \psi}{\longrightarrow} \Gamma^{\lambda_{1}}(M \otimes N) \otimes \ldots \otimes \Gamma^{\lambda_{m}}(M \otimes N) \stackrel{\nabla}{\longrightarrow} \Gamma^{d}(M \otimes N),
\end{aligned}
$$

where the first map permutes tensor factors and the last map is multiplication in the bialgebra $\Gamma(M \otimes N)$.

Let us write $\Gamma^{\lambda}: \mathcal{P}_{\mathbb{k}} \rightarrow \mathcal{P}_{\mathbb{k}}$ to denote the tensor product of functors

$$
\Gamma^{\lambda}:=\Gamma^{\lambda_{1}} \otimes \cdots \otimes \Gamma^{\lambda_{m}}
$$

defined in the same way as (2.10). Then it follows from Lemma 2.5 that the maps $\psi^{\lambda}(M, N)$ induce a natural transformation

$$
\psi^{\lambda}: \Gamma^{\lambda} \otimes \Gamma^{\lambda} \rightarrow \Gamma^{\lambda}(-\otimes-)
$$

of bifunctors $\mathcal{P}_{\mathbb{k}} \times \mathcal{P}_{\mathbb{k}} \rightarrow \mathcal{P}_{\mathbb{k}}$.

The following lemma is a special case of [12, Proposition III.2.6] which describes the relationship between $\psi$-maps and standard homomorphisms.

Lemma $5.3([12])$. Suppose $\lambda \in \Lambda_{d}^{+}(\mathbb{N})$, and set $q=l(\lambda)$. Given a pair $U, V$ of free $\mathbb{k}$-modules of finite rank, the following diagram is commutative

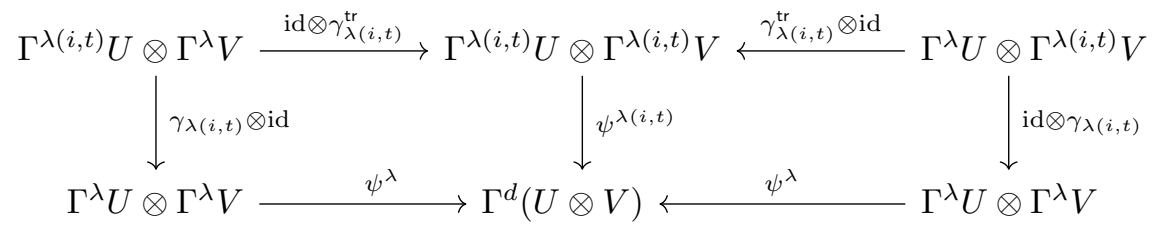

for any $i \in[1, q-1]$ and $t \in\left[1, \lambda_{i+1}\right]$.

Recalling the total order $\preceq$ on $\Lambda_{d}^{+}(\mathbb{N})$ from Definition 2.2 , write $\lambda^{+}$to denote the immediate successor of a partition $\lambda$ and set $(d)^{+}:=\infty$. The Cauchy filtration is then defined as the chain

$$
0=\mathcal{F}_{\infty} \subset \mathcal{F}_{(d)} \subset \cdots \subset \mathcal{F}_{(1, \ldots, 1)}=\Gamma^{d}(M \otimes N)
$$

where $\mathcal{F}_{\lambda}:=\sum_{\mu \succeq \lambda} \operatorname{Im}\left(\psi^{\lambda}\right)$.

The following result describes the factors of this filtration.

Theorem 5.4 ([12, Theorem III.2.7]). Let $U, V$ be free $\mathbb{k}$-modules of finite rank. Then for each $\lambda \in \Lambda_{d}^{+}(\mathbb{N})$, the map $\psi^{\lambda}$ induces an isomorphism

$$
\bar{\psi}^{\lambda}: W_{\lambda}(U) \otimes W_{\lambda}(V) \stackrel{\sim}{\longrightarrow} \mathcal{F}_{\lambda} / \mathcal{F}_{\lambda^{+}}
$$

which makes the following diagram commutative:

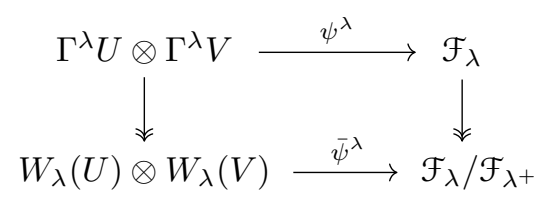


Hence, the associated graded module of the Cauchy filtration is

$$
\bigoplus_{\lambda \in \Lambda_{d}^{+}(\mathbb{N})} W_{\lambda}(U) \otimes W_{\lambda}(V)
$$

Proof. We recall the proof from [12]. It follows by definition that $W_{\lambda}(U) \otimes W_{\lambda}(V)$ is the quotient of $\Gamma^{\lambda} U \otimes \Gamma^{\lambda} V$ by the submodule $\square_{\lambda}(U) \otimes \Gamma^{\lambda} V+\Gamma^{\lambda} U \otimes \square_{\lambda}(V)$. Hence, by Lemma 5.3 we have

$$
\square_{\lambda}(U) \otimes \Gamma^{\lambda} V+\Gamma^{\lambda} U \otimes \square_{\lambda}(V) \subset \operatorname{Im}\left(\psi^{\lambda(i, t)}\right) \subset \mathcal{F}_{\lambda^{+}},
$$

since $\lambda(i, t)>\lambda$. This proves the existence of the induced map $\bar{\psi}^{\lambda}$ satisfying the given commutative square. It is clear that $\bar{\psi}^{\lambda}$ is surjective. Comparing the ranks of $\Gamma^{d}(U \otimes V)$ and $\bigoplus_{\lambda \in \Lambda_{d}^{+}(\mathbb{N})} W_{\lambda}(U) \otimes W_{\lambda}(V)$ shows that $\bar{\psi}^{\lambda}$ must be an isomorphism for each $\lambda$.

Given free $\mathbb{k}$-modules $U, V \in \mathcal{P}_{\mathbb{k}}$ with finite ordered bases $\left\{x_{b}\right\}_{b \in \mathcal{B}}$ and $\left\{y_{c}\right\}_{c \in \mathcal{C}}$, respectively, let $\mathcal{F}_{\lambda}^{\prime} \subset \Gamma^{d}(U \otimes V)$ denote the $\mathbb{k}$-submodule generated by

$$
\left\{\psi^{\lambda}\left(x_{S} \otimes y_{T}\right) \mid S \in \mathrm{St}_{\lambda}(\mathcal{B}), T \in \mathrm{St}_{\lambda}(\mathcal{C})\right\}
$$

where $\mathcal{F}_{\lambda}^{\prime}$ is nonzero only if $l(\lambda) \leq \min (\sharp \mathcal{B}, \sharp \mathcal{C})$.

Corollary 5.5. For each $\lambda \in \Lambda_{d}^{+}(\mathbb{N})$, the $\mathbb{k}$-submodule $\mathcal{F}_{\lambda}^{\prime} \subset \Gamma^{d}(U \otimes V)$ is free, and there is a corresponding decomposition:

$$
\Gamma^{d}(U \otimes V)=\bigoplus_{\lambda} \mathcal{F}_{\lambda}^{\prime}, \quad \text { such that } \quad \mathcal{F}_{\lambda}=\bigoplus_{\mu \geq \lambda} \mathcal{F}_{\mu}^{\prime} \quad \text { for all } \lambda \in \Lambda_{d}^{+}(\mathbb{N}) .
$$

Proof. Suppose $\lambda \in \Lambda_{d}^{+}(\mathbb{N})$, and set $\mathcal{T}=\mathrm{St}_{\lambda}(\mathcal{B}) \times \mathrm{St}_{\lambda}(\mathcal{C})$. By Proposition 5.2, $\left\{\bar{x}_{S} \otimes \bar{y}_{T} \mid(S, T) \in \mathcal{T}\right\}$ forms a basis of $W_{\lambda}(U) \otimes W_{\lambda}(V)$. So

$$
\left\{\bar{\psi}^{\lambda}\left(x_{S} \otimes y_{T}\right) \mid(S, T) \in \mathcal{T}\right\}
$$

gives a basis for $\mathcal{F}_{\lambda} / \mathcal{F}_{\lambda^{+}}$by Theorem 5.4. This shows that the subset

$$
\left\{\psi^{\lambda}\left(x_{S} \otimes y_{T}\right) \mid(S, T) \in \mathcal{T}\right\} \subset \Gamma^{d}(U \otimes V)
$$

is linearly independent. Thus $\mathcal{F}_{\lambda}^{\prime}$ is a free $\mathbb{k}$-submodule. It is also clear that $\mathcal{F}_{\lambda}=$ $\mathcal{F}_{\lambda^{+}} \oplus \mathcal{F}_{\lambda}^{\prime}$, and the required decompositions follow by induction.

5.4. Bimodule filtrations. In the remainder of this section, we fix a set $\left\{J_{1}^{\prime}, \ldots, J_{r}^{\prime}\right\}$ of nonzero free $\mathbb{k}$-submodules, $J_{i}^{\prime} \subset J$, such that setting

$$
J_{j}:=\bigoplus_{1 \leq i \leq j} J_{i}^{\prime} \quad \text { for } j \in[1, r]
$$

yields a chain

$$
0=J_{0} \subset J_{1} \subset \cdots \subset J_{r}=J
$$

of $(A, B)$-bimodules.

Recalling the notation (3.4), we then have for each $\mu \in \Lambda_{d}(r)$ the following $\mathbb{k}$-submodules of $\Gamma^{d} J$ :

$$
J_{\mu}^{\prime}=\left\langle J_{\otimes \mu}^{\prime}\right\rangle^{\mathfrak{S}_{d}}, \quad J_{\mu}=\left\langle J_{\otimes \mu}\right\rangle^{\mathfrak{S}_{d}}
$$

Note first that $J_{\mu}$ is a $\Gamma^{d}(A \otimes B)$-submodule of $\Gamma^{d} J$, and hence a $\left(\Gamma^{d} A, \Gamma^{d} B\right)$ bimodule. It is also not difficult to check that there is a decomposition of $J^{\otimes d}$ into free $\mathbb{k}$-submodules

$$
J^{\otimes d}=\bigoplus_{\mu \in \Lambda_{d}(r)}\left\langle J_{\otimes \mu}^{\prime}\right\rangle
$$


By taking $\mathfrak{S}_{d}$-invariants on both sides, we thus obtain the following decomposition

$$
\Gamma^{d} J=\bigoplus_{\mu \in \Lambda_{d}(r)}\left\langle J_{\otimes \mu}^{\prime}\right\rangle \cap \Gamma^{d} J=\bigoplus_{\mu \in \Lambda_{d}(r)} J_{\mu}^{\prime} .
$$

Next recall that the dominance order on $\Lambda_{d}(r)$ is the partial order defined by setting $\mu \unlhd \nu$ if

$$
\sum_{i \leq j} \mu_{i} \leq \sum_{i \leq j} \nu_{i} \text { for } j \in[1, r] .
$$

Notice that $J_{\mu} \subset J_{\nu}$ if and only if $\mu \unrhd \nu$. We further have $J_{\nu}=J_{\nu}^{\prime} \oplus \sum_{\mu \triangleright \nu} J_{\mu}$, and it follows by induction that

$$
J_{\nu}=\bigoplus_{\mu \unrhd \nu} J_{\mu}^{\prime}
$$

for all $\nu \in \Lambda_{d}(r)$, which generalizes the decomposition (5.6) of $\Gamma^{d} J$.

Consider the map $\nabla: \Gamma^{\mu} J \rightarrow \Gamma^{d} J$ given by $r$-fold (outer) multiplication in $\Gamma(J)$, for some $\mu \in \Lambda_{d}(r)$. Note that the restriction

$$
\nabla^{\mu}: \Gamma^{(\mu)}\left(J_{1}, \ldots, J_{r}\right) \rightarrow \Gamma^{d} J \quad\left(\operatorname{resp} .{ }^{\prime} \nabla^{\mu}: \Gamma^{(\mu)}\left(J_{1}^{\prime}, \ldots, J_{r}^{\prime}\right) \rightarrow \Gamma^{d} J\right)
$$

is a $\left(\Gamma^{d} A, \Gamma^{d} B\right)$-bimodule (resp. $\mathbb{k}$-module) homomorphism.

Lemma 5.6. Suppose $\nu \in \Lambda_{d}(r)$. Then

(1) $J_{\nu}^{\prime}=\operatorname{Im}^{\prime} \nabla^{\nu}$

(2) ${ }^{\prime} \nabla^{\nu}: \Gamma^{(\nu)}\left(J_{1}^{\prime}, \ldots, J_{r}^{\prime}\right) \stackrel{\sim}{\longrightarrow} J_{\nu}^{\prime}$ is an isomorphism of $\mathbb{k}$-modules,

(3) $J_{\nu}=\sum_{\mu \unrhd \nu} \operatorname{Im} \nabla^{\mu}$, summing over $\mu \in \Lambda_{d}(r)$.

Proof. For each $\mu \in \Lambda_{d}(r)$, write $M_{\mu}, M_{\mu}^{\prime}$ to denote the images of $\Gamma^{(\mu)}\left(J_{1}, \ldots, J_{r}\right)$ and $\Gamma^{(\mu)}\left(J_{1}^{\prime}, \ldots, J_{r}^{\prime}\right)$, respectively, under the map $\nabla^{\mu}: \Gamma^{\mu} J \rightarrow \Gamma^{d} J$. It is then clear from the definitions that $M_{\mu}^{\prime} \subset J_{\mu}^{\prime}$ and similarly $M_{\mu}=\operatorname{Im} \nabla^{\mu} \subset J_{\mu}$, for all $\mu$.

It follows inductively from the isomorphism (2.7) that there is a decomposition

$$
\Gamma^{d} J=\Gamma^{d}\left(J_{1}^{\prime} \oplus \cdots \oplus J_{r}^{\prime}\right)=\bigoplus_{\mu \in \Lambda_{d}(r)} M_{\mu}^{\prime} \subset \bigoplus_{\mu \in \Lambda_{d}(r)} J_{\mu}^{\prime}
$$

It thus follows from (5.6) that $J_{\mu}^{\prime}=M_{\mu}^{\prime} \cong \Gamma^{(\mu)}\left(J_{1}^{\prime}, \ldots, J_{r}^{\prime}\right)$ which shows (1) and (2). Since $J_{\mu} \subset J_{\nu}$ whenever $\mu \unrhd \nu$, it follows from (5.7) that

$$
J_{\nu}=\bigoplus_{\mu \unrhd \nu} M_{\mu}^{\prime} \subset \sum_{\mu \unrhd \nu} M_{\mu} \subset \sum_{\mu \unrhd \nu} J_{\mu} \subset J_{\nu}
$$

showing (3).

Recall the lexicographic ordering $\leq$ on $\Lambda_{d}(r)$ from Definition 2.2, and notice that there is a chain of $\left(\Gamma^{d} A, \Gamma^{d} B\right)$-sub-bimodules

$$
0 \subset \Gamma^{d}\left(J_{1}\right)=J_{\geq(d, 0, \ldots, 0)} \subset \cdots \subset J_{\geq \nu} \subset \cdots \subset J_{\geq(0, \ldots, 0, d)}=\Gamma^{d} J
$$

where $J_{\geq \nu}:=\sum_{\mu \geq \nu} J_{\mu}$ for each $\nu \in \Lambda_{d}(r)$. Since the lexicographic ordering refines the dominance order, it follows from (5.7) that

$$
J_{\geq \nu}=J_{>\nu} \oplus J_{\nu}^{\prime}
$$

for all $\nu$. Thus

$$
J_{\geq \nu}=\sum_{\mu \geq \nu} \operatorname{Im}\left(\nabla^{\mu}\right)
$$

by the preceding lemma. This allows us to describe the quotients $J_{\geq \nu} / J_{>\nu}$ as follows. 
Proposition 5.7. Let $\nu \in \Lambda_{d}(r)$. Then $\nabla^{\nu}$ induces an isomorphism

$$
\bar{\nabla}^{\nu}: \Gamma^{(\nu)}\left(J_{1} / J_{0}, \ldots, J_{r} / J_{r-1}\right) \cong J_{\geq \nu} / J_{>\nu}
$$

which yields a commutative square of $\left(\Gamma^{d} A, \Gamma^{d} B\right)$-bimodule homomorphisms

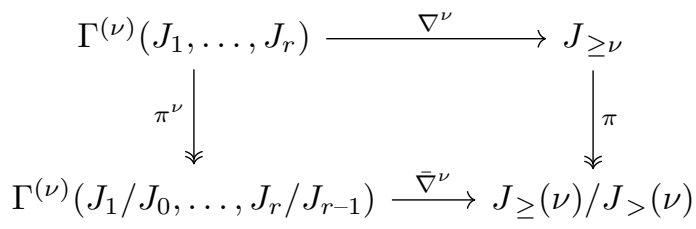

where $\pi^{\nu}$ denotes the tensor product of functorial maps $\Gamma^{\nu_{j}}\left(\pi_{j}\right)$ associated to the projections, $\pi_{j}: J_{j} \rightarrow J_{j} / J_{j-1}$, for $j=1, \ldots, r$, and where $\pi$ is also projection.

Proof. We first verify that $\operatorname{ker} \pi^{\nu} \subset J_{>\nu}$ in order to show the existence of the map $\bar{\nabla}^{\nu}$ satisfying the above diagram. If $1 \leq j \leq r$, consider the $\left(\Gamma^{d} A, \Gamma^{d} B\right)$-subbimodule

$$
K_{j}:=\Gamma^{\left(\nu^{1}\right)}\left(J_{1}, \ldots, J_{r}\right) \otimes \operatorname{ker} \Gamma^{\nu_{j}}\left(\pi_{j}\right) \otimes \Gamma^{\left(\nu^{2}\right)}\left(J_{1}, \ldots, J_{r}\right)
$$

where $\nu^{1}=\left(\nu_{1}, \ldots, \nu_{j-1}, 0, \ldots, 0\right)$ and $\nu^{2}=\left(0, \ldots, 0, \nu_{j+1}, \ldots, \nu_{r}\right)$. Then $\operatorname{ker} \pi^{\nu}=$ $\sum_{j=1}^{r} K_{j}$, and we must show that $K_{j} \subset J_{>\nu}$ for all $j$.

Now $K_{j}=0$, if either $j=1$ or $\nu_{j}=0$. If $K_{j} \neq 0$ and $1 \leq t \leq \nu_{j}$, let $\nu(j, t) \in \Lambda_{d}(r)$ be defined as in (5.1). Since $\nu(j, 1)>\nu$, it suffices to show that $\nabla^{\nu}\left(K_{j}\right) \subset \operatorname{Im} \nabla^{\nu(j, 1)}$ for all such $j$. The fact that $\nu$ and $\nu(j, 1)$ are equal except for entries in the $j$-th and $(j-1)$-st positions allows us to simplify to the case $r=2$.

So we may assume $\nu=\left(\nu_{1}, \nu_{2}\right)$. Then for $j=2$, we have $\nu(2,1)=\left(\nu_{1}+1, \nu_{2}-1\right)$. In this case $K_{2}=\operatorname{ker}\left(\pi^{\nu}\right)$, and it follows by Lemma 3.5 that

$$
K_{2}=\Gamma^{\nu_{1}} J_{1} \otimes J_{\left(1, \nu_{2}-1\right)} \subset \Gamma^{(\nu)}\left(J_{1}, J_{2}\right) .
$$

Notice by Lemma 5.6 that $J_{\left(1, \nu_{2}-1\right)}$ is equal to the image of the map

$$
\nabla^{\left(1, \nu_{2}-1\right)}: J_{1} \otimes \Gamma^{\nu_{2}-1}\left(J_{2}\right) \rightarrow \Gamma^{\nu_{2}}\left(J_{2}\right) .
$$

By associativity of multiplication in $\Gamma(J)$, we also have a commutative diagram

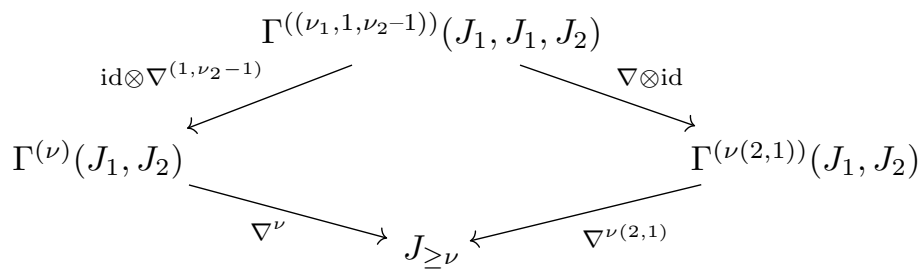

It follows that $\nabla^{\nu}\left(K_{2}\right) \subset \operatorname{Im} \nabla^{\nu(2,1)}$, which shows the existence of $\bar{\nabla}^{\nu}$. To complete the proof, note that the restriction $\left.\pi^{\nu}\right|_{\Gamma^{(\nu)}\left(J_{1}^{\prime}, \ldots, J_{r}^{\prime}\right)}$ is a $\mathbb{k}$-module isomorphism. The map $\left.\left(\pi \circ \nabla^{\nu}\right)\right|_{\Gamma^{(\nu)}\left(J_{1}^{\prime}, \ldots, J_{r}^{\prime}\right)}$ is also a $\mathbb{k}$-module isomorphism by Lemma 5.6. It follows that $\bar{\nabla}^{\nu}$ is an isomorphism by commutativity.

5.5. Multitableaux. Suppose $\left\{\mathcal{B}_{j}\right\}_{j \in[1, r]}$ is a collection of finite totally ordered sets, and let $\boldsymbol{\lambda} \in \Lambda_{d}^{+}(\mathbb{N})^{r}$ be an $r$-multipartition. Elements of the set

$$
\operatorname{Tab}_{\boldsymbol{\lambda}}\left(\mathcal{B}_{1}, \ldots, \mathcal{B}_{r}\right):=\operatorname{Tab}_{\lambda^{(1)}}\left(\mathcal{B}_{1}\right) \times \cdots \times \operatorname{Tab}_{\lambda^{(r)}}\left(\mathcal{B}_{r}\right) .
$$

are called multitableaux of shape $\boldsymbol{\lambda}$ (or $\boldsymbol{\lambda}$-multitableaux).

We say that a $\boldsymbol{\lambda}$-multitableau, $\mathbf{T}=\left(\mathrm{T}^{(1)}, \ldots, \mathrm{T}^{(r)}\right)$, is standard if each component $\mathrm{T}^{(j)}$ is a standard $\lambda^{(j)}$-tableau. The subset of standard $\boldsymbol{\lambda}$-multitableaux is denoted

$$
\operatorname{St}_{\boldsymbol{\lambda}}\left(\mathcal{B}_{*}\right)=\operatorname{St}_{\boldsymbol{\lambda}}\left(\mathcal{B}_{1}, \ldots, \mathcal{B}_{r}\right) .
$$


If $\left(n_{1}, \ldots, n_{r}\right) \in \mathbb{N}^{r}$ is the sequence of integers with $n_{j}:=\sharp \mathcal{B}_{j}$ for all $j$, then it follows from (5.3) that $\operatorname{St}_{\boldsymbol{\lambda}}\left(\mathcal{B}_{*}\right)$ is non-empty if and only if $\boldsymbol{\lambda}$ belongs to the subset $\Lambda_{d}^{+}\left(n_{1}, \ldots, n_{r}\right) \subset \Lambda_{d}^{+}(\mathbb{N})^{r}$. In this case, we write $\mathbf{T}^{\boldsymbol{\lambda}}=\mathbf{T}^{\boldsymbol{\lambda}}\left(\mathcal{B}_{*}\right)$ to denote the standard $\boldsymbol{\lambda}$-multitableau

$$
\mathbf{T}^{\boldsymbol{\lambda}}:=\left(\mathrm{T}^{\lambda^{(1)}}, \ldots, \mathrm{T}^{\lambda^{(r)}}\right) .
$$

Suppose $\nu=\left(\nu_{1}, \ldots, \nu_{r}\right) \in \Lambda_{d}(r)$. There is a corresponding $r$-multipartition $(\nu):=\left(\left(\nu_{1}\right),\left(\nu_{2}\right), \ldots,\left(\nu_{r}\right)\right) \in \Lambda_{d}^{+}(\mathbb{N})^{r}$. For any $m \in \mathbb{N}$, let us write $\left(1^{m}\right):=$ $(1, \ldots, 1) \in \Lambda_{m}^{+}(\mathbb{N})$, and set $\left(1^{0}\right)=0$. Then we also have an element

$$
(\nu)^{\prime}:=\left(\left(1^{\nu_{1}}\right),\left(1^{\nu_{2}}\right), \ldots,\left(1^{\nu_{r}}\right)\right) \in \Lambda_{\nu}^{+}(\mathbb{N}) .
$$

Recalling the total order $\preceq$ from Definition 2.4 , notice that $(\nu)^{\prime} \preceq \boldsymbol{\lambda} \preceq(\nu)$ for all $\boldsymbol{\lambda} \in \Lambda_{\nu}^{+}(\mathbb{N})$. We also write $\boldsymbol{\lambda}^{+}$to denote the immediate successor of any $\boldsymbol{\lambda} \in \Lambda_{d}^{+}(\mathbb{N})^{r}$ and set $((d))^{+}=\infty$.

5.6. Generalized Weyl modules. Given $\boldsymbol{\lambda} \in \Lambda_{d}^{+}(\mathbb{N})^{r}$ and projective modules $M_{j} \in \mathcal{P}_{\mathbb{k}}$ for $j \in[1, r]$, we will use the notation

$$
\Gamma^{\boldsymbol{\lambda}}\left(M_{*}\right):=\bigotimes_{j} \Gamma^{\lambda^{(j)}} M_{j}, \quad W_{\boldsymbol{\lambda}}\left(M_{*}\right):=\bigotimes_{j} W_{\lambda^{(j)}} M_{j}
$$

in what follows. The outer tensor product $-\otimes-$, defined in Section 2.7, yields corresponding functors $\Gamma^{\boldsymbol{\lambda}}, W_{\boldsymbol{\lambda}}: \mathcal{P}_{\mathbb{k}}^{\times r} \rightarrow \mathcal{P}_{\mathbb{k}}$ defined by

$$
\Gamma^{\boldsymbol{\lambda}}:=\Gamma^{\lambda^{(1)}} \otimes \cdots \otimes \Gamma^{\lambda^{(r)}} \quad \text { and } W_{\boldsymbol{\lambda}}:=W_{\lambda^{(1)}} \otimes \cdots \otimes W_{\lambda^{(r)}} .
$$

Since Weyl modules are quotients of divided powers, it follows that there is a natural projection $\pi: \Gamma^{\boldsymbol{\lambda}} \rightarrow W_{\boldsymbol{\lambda}}$.

Suppose $V_{1}, \ldots, V_{r} \in \mathcal{P}_{\mathbb{k}}$ are free $\mathbb{k}$-modules, and suppose $\left\{x_{b}^{(j)}\right\}_{b \in \mathcal{B}_{j}}$ is a finite ordered basis of $V_{j}$ for each $j \in[1, r]$. Given a multitableau $\mathbf{T} \in \operatorname{Tab}_{\boldsymbol{\lambda}}\left(\mathcal{B}_{1}, \ldots, \mathcal{B}_{r}\right)$, there is a corresponding element

$$
x_{\mathbf{T}}:=\bigotimes_{j} x_{\mathbf{T}^{(j)}}^{(j)} \in \Gamma^{\boldsymbol{\lambda}}\left(V_{*}\right)
$$

whose image in $W_{\boldsymbol{\lambda}}\left(V_{*}\right)$ is denoted $\bar{x}_{\mathbf{T}}:=\pi\left(x_{\mathbf{T}}\right)$. The next result follows easily from Proposition 5.2.

Lemma 5.8. Let $\boldsymbol{\lambda} \in \Lambda_{d}^{+}(\mathbb{N})^{r}$ be an $r$-multipartition, and let $V_{1}, \ldots, V_{r}$ be free $\mathbb{k}$ modules with bases as above. The set of images $\left\{\bar{x}_{\mathbf{T}} \mid \mathbf{T} \in \operatorname{St}_{\boldsymbol{\lambda}}\left(\mathcal{B}_{1}, \ldots, \mathcal{B}_{r}\right)\right\}$ forms a basis of the free $\mathbb{k}$-module $W_{\boldsymbol{\lambda}}\left(V_{*}\right)$ parametrized by standard $\boldsymbol{\lambda}$-multitableaux. In particular, we have $W_{\boldsymbol{\lambda}}\left(V_{*}\right)=0$ unless $\boldsymbol{\lambda} \in \Lambda_{d}^{+}\left(\sharp \mathcal{B}_{1}, \ldots, \sharp \mathcal{B}_{r}\right)$.

Suppose $\nu \in \Lambda_{d}(r)$ and fix some projective modules $M_{j}, N_{j} \in \mathcal{P}_{\mathbb{k}}$ for $j \in[1, r]$. Using notation similar to the above, we write

$$
\Gamma^{(\nu)}\left(M_{*} \otimes N_{*}\right):=\bigotimes_{j} \Gamma^{\nu_{j}}\left(M_{j} \otimes N_{j}\right)
$$

Given $\boldsymbol{\lambda} \in \Lambda_{\nu}^{+}(\mathbb{N})$, we then define a map

$$
\psi^{\boldsymbol{\lambda}}: \Gamma^{\boldsymbol{\lambda}}\left(M_{*}\right) \otimes \Gamma^{\boldsymbol{\lambda}}\left(N_{*}\right) \rightarrow \Gamma^{(\nu)}\left(M_{*} \otimes N_{*}\right)
$$

via the composition

$$
\begin{aligned}
\left\{\bigotimes_{j} \Gamma^{\lambda^{(j)}} M_{j}\right\} & \otimes\left\{\bigotimes_{j} \Gamma^{\lambda^{(j)}} N_{j}\right\} \\
& \cong \bigotimes_{j}\left\{\Gamma^{\lambda^{(j)}}\left(M_{j}\right) \otimes \Gamma^{\lambda^{(j)}}\left(N_{j}\right)\right\} \stackrel{\psi \otimes \ldots \otimes \psi}{\longrightarrow} \bigotimes_{j} \Gamma^{\nu_{j}}\left(M_{j} \otimes N_{j}\right) .
\end{aligned}
$$

Note that if $M_{j} \in A$-mod and $N_{j} \in B$-mod for all $j$, then $\psi^{\boldsymbol{\lambda}}$ is a homomorphism of $\left(\Gamma^{d} A, \Gamma^{d} B\right)$-bimodules by Lemma 2.5.1. 
5.7. Generalized Cauchy filtrations of bimodules. Fix a chain $\left(J_{j}\right)_{j \in[0, r]}$ of ( $A, B)$-bimodules. For each $j \in[1, r]$, suppose there exists an isomorphism

$$
\alpha_{j}: J_{j} / J_{j-1} \stackrel{\sim}{\longrightarrow} U_{j} \otimes V_{j}
$$

of $(A, B)$-bimodules for some $U_{j} \in A$-mod and $V_{j} \in B$-mod. Assume for all $j$ that $U_{j}$ and $V_{j}$ are free as $\mathbb{k}$-modules, with finite ordered bases $\left\{x_{b}^{(j)}\right\}_{b \in \mathcal{B}_{j}}$ and $\left\{y_{c}^{(j)}\right\}_{c \in \mathcal{C}_{j}}$, respectively. Assume further that $\left\{J_{j}^{\prime}\right\}_{j \in[r]}$ is any collection of free $\mathbb{k}$-submodules of $J_{r}$ such that (5.5) holds.

We first define a filtration of $\Gamma^{(\nu)}\left(U_{*} \otimes V_{*}\right)$ for some fixed weight $\nu \in \Lambda_{d}(r)$. For each $r$-multipartition $\boldsymbol{\lambda} \in \Lambda_{\nu}^{+}(\mathbb{N})^{r}$, let us write

$$
\mathcal{F}_{\boldsymbol{\lambda},(\nu)}:=\sum_{\boldsymbol{\lambda} \leq \boldsymbol{\mu} \leq(\nu)} \mathcal{F}_{\mu^{(1)}}\left(U_{1}, V_{1}\right) \otimes \cdots \otimes \mathcal{F}_{\mu^{(r)}}\left(U_{r}, V_{r}\right)
$$

which is a sum of sub-bimodules of $\Gamma^{(\nu)}\left(U_{*} \otimes V_{*}\right)$. It follows that there is a chain of sub-bimodules:

$$
0=: \mathcal{F}_{(\nu)^{+},(\nu)} \subset \mathcal{F}_{(\nu),(\nu)} \subset \cdots \subset \mathcal{F}_{(\nu)^{\prime},(\nu)}=\Gamma^{(\nu)}\left(U_{*} \otimes V_{*}\right) .
$$

Recalling (5.10), notice that for each $\boldsymbol{\lambda} \in \Lambda_{\nu}^{+}(\mathbb{N})^{r}$ we have

$$
\mathcal{F}_{\boldsymbol{\lambda},(\nu)}=\sum_{\boldsymbol{\lambda} \preceq \boldsymbol{\mu} \preceq(\nu)} \operatorname{Im}\left(\psi^{\boldsymbol{\mu}}\right) .
$$

Note also that $\mathcal{F}_{\boldsymbol{\lambda},(\nu)}$ contains the $\mathbb{k}$-submodule

$$
\mathcal{F}_{\lambda}^{\prime}:=\bigotimes \mathcal{F}_{\lambda^{(j)}}^{\prime}\left(U_{j}, V_{j}\right)
$$

It then follows by Corollary 5.5 that $\mathcal{F}_{\boldsymbol{\lambda}}^{\prime}$ is a free $\mathbb{k}$-submodule, with the set

$$
\left\{\psi^{\boldsymbol{\lambda}}\left(x_{\mathbf{S}} \otimes y_{\mathbf{T}}\right) \mid \mathbf{S} \in \mathbf{S t}_{\boldsymbol{\lambda}}\left(\mathcal{B}_{1}, \ldots, \mathcal{B}_{r}\right), \mathbf{T} \in \mathbf{S t}_{\boldsymbol{\lambda}}\left(\mathcal{C}_{1}, \ldots, \mathcal{C}_{r}\right)\right\}
$$

as a basis.

Proposition 5.9. Suppose $\nu \in \Lambda_{d}(r)$. Then for each $\boldsymbol{\lambda} \in \Lambda_{\nu}^{+}(\mathbb{N})$, the map

$$
\psi^{\boldsymbol{\lambda}}: \Gamma^{\boldsymbol{\lambda}}\left(U_{*}\right) \otimes \Gamma^{\boldsymbol{\lambda}}\left(V_{*}\right) \rightarrow \mathcal{F}_{\boldsymbol{\lambda},(\nu)}
$$

induces an isomorphism

$$
\bar{\psi}^{\boldsymbol{\lambda}}: \mathcal{F}_{\boldsymbol{\lambda},(\nu)} / \mathcal{F}_{\boldsymbol{\lambda}^{+},(\nu)} \stackrel{\sim}{\longrightarrow} W_{\boldsymbol{\lambda}}\left(U_{*}\right) \otimes W_{\boldsymbol{\lambda}}\left(V_{*}\right)
$$

of bimodules. We also have decompositions

$$
\Gamma^{(\nu)}\left(U_{*} \otimes V_{*}\right)=\bigoplus_{\boldsymbol{\lambda} \in \Lambda_{\nu}^{+}(\mathbb{N})} \mathcal{F}_{\boldsymbol{\lambda}}^{\prime}, \quad \mathcal{F}_{\boldsymbol{\lambda},(\nu)}=\bigoplus_{\boldsymbol{\lambda} \preceq \boldsymbol{\mu} \preceq(\nu)} \mathcal{F}_{\boldsymbol{\mu}}^{\prime}
$$

into free $\mathbb{k}$-submodules.

We now wish to lift the filtrations (5.12), for varying $\nu$, to a single filtration of $\Gamma^{d} J$, with $J=J_{r}$ as above. First note that there is an isomorphism

$$
\phi_{\nu}: J_{\geq \nu} / J_{>\nu} \stackrel{\sim}{\longrightarrow} \Gamma^{(\nu)}\left(U_{*} \otimes V_{*}\right)
$$

satisfying the following commutative triangle of $\left(\Gamma^{d} A, \Gamma^{d} B\right)$-bimodule isomorphisms:

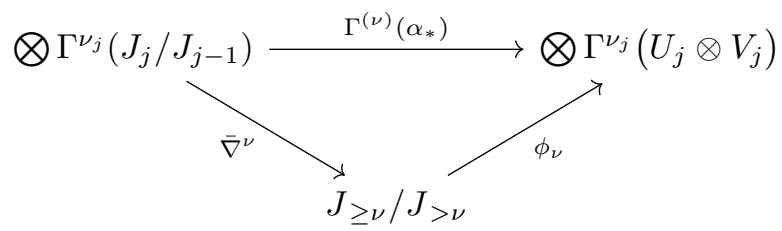


where $\Gamma^{(\nu)}\left(\alpha_{*}\right)=\otimes \Gamma^{\nu_{j}}\left(\alpha_{j}\right)$ is a tensor product of isomorphisms induced by the maps (5.11) and $\bar{\nabla}^{\nu}$ is defined in Proposition 5.7. We then have a surjective map

$$
\hat{\phi}_{\nu}: J_{\geq \nu} \rightarrow \Gamma^{(\nu)}\left(U_{*} \otimes V_{*}\right)
$$

obtained by composing $\phi_{\nu}$ with the projection $\pi: J_{\geq \nu} \rightarrow J_{\geq \nu} / J_{>\nu}$.

Definition 5.10. Suppose $\boldsymbol{\lambda} \in \Lambda_{d}^{+}(\mathbb{N})^{r}$ and set $\nu=|\boldsymbol{\lambda}|$. Then define $\mathcal{J}_{\boldsymbol{\lambda}}$ to be the sub-bimodule of $J_{\geq \nu}$, corresponding to the inverse image of $\mathcal{F}_{\boldsymbol{\lambda},(\nu)}$ under the map $\phi_{\nu}$ considered above. The generalized Cauchy filtration of $\Gamma^{d} J$ is then defined as the chain

$$
0=\mathcal{J}_{\infty} \subset \mathcal{J}_{((d))} \subset \cdots \subset \mathcal{J}_{\boldsymbol{\lambda}^{+}} \subset \mathcal{J}_{\boldsymbol{\lambda}} \subset \cdots \subset \mathcal{J}_{\left(\left(1^{d}\right)\right)}=\Gamma^{d} J
$$

of $\left(\Gamma^{d} A, \Gamma^{d} B\right)$-bimodules parametrized by multipartitions $\boldsymbol{\lambda} \in \Lambda_{d}^{+}(\mathbb{N})^{r}$.

We next define a decomposition of $\Gamma^{d} J$ via certain $\mathbb{k}$-submodules, $\partial_{\boldsymbol{\lambda}}^{\prime} \subset \partial_{\boldsymbol{\lambda}}$. Recall from (5.5) that $J_{j}=J_{j}^{\prime} \oplus J_{j-1}$, for all $j$. For each $j \in[1, r]$, let

$$
\alpha_{j}^{\prime}: J_{j}^{\prime} \stackrel{\sim}{\rightarrow} U_{j} \otimes V_{j}
$$

denote the isomorphism defined via the composition

$$
J_{j}^{\prime} \hookrightarrow J_{j} \rightarrow J_{j} / J_{j-1} \stackrel{\alpha_{j}}{\longrightarrow} U_{j} \otimes V_{j} .
$$

Similar to (5.15), there is a resulting $\mathbb{k}$-module isomorphism

$$
\phi_{\nu}^{\prime}: J_{\nu}^{\prime} \stackrel{\sim}{\rightarrow} \Gamma^{(\nu)}\left(U_{*} \otimes V_{*}\right)
$$

satisfying the following commutative triangle of isomorphisms:

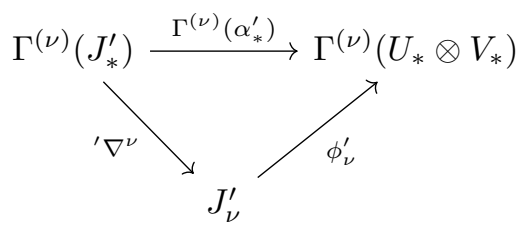

where

$$
\Gamma^{(\nu)}\left(\alpha_{*}^{\prime}\right):=\bigotimes \Gamma^{\nu_{j}}\left(\alpha_{j}^{\prime}\right)
$$

and where ${ }^{\prime} \nabla^{\nu}$ is restriction of $r$-fold multiplication as in Lemma 5.6.(i). We write

$$
\mathcal{J}_{\lambda}^{\prime}:=\left(\phi_{\nu}^{\prime}\right)^{-1}\left(\mathcal{F}_{\lambda}^{\prime}\right)
$$

to denote the inverse image of $\mathcal{F}_{\boldsymbol{\lambda}}^{\prime}$ under $\phi_{\nu}^{\prime}$.

Lemma 5.11. There exist decompositions into free $\mathbb{k}$-submodules

$$
\Gamma^{d} J=\bigoplus_{\boldsymbol{\lambda} \in \Lambda_{d}^{+}(\mathbb{N})^{r}} J_{\boldsymbol{\lambda}}^{\prime}, \quad \text { and } \quad \mathcal{J}_{\boldsymbol{\lambda}}=\bigoplus_{\boldsymbol{\lambda} \preceq \boldsymbol{\mu} \prec \infty} \mathcal{J}_{\boldsymbol{\mu}}^{\prime} \quad \text { for each } \boldsymbol{\lambda} .
$$

Proof. It follows by definition from (5.15) and (5.17) that $\phi_{\nu}^{\prime}$ can be obtained from $\hat{\phi}$ by restriction. In particular, we have a commutative diagram:

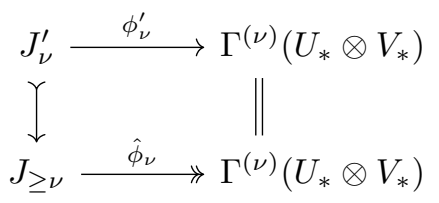

Since $J_{\geq \nu}=J_{>\nu} \oplus J_{\nu}^{\prime}$ by (5.8), we further have a decomposition

$$
\hat{\phi}_{\nu}^{-1}(N)=J_{>\nu} \oplus\left(\phi_{\nu}^{\prime}\right)^{-1}(N)
$$


for any $\mathbb{k}$-submodule $N \subset \Gamma^{(\nu)}\left(U_{*} \otimes V_{*}\right)$. If we set $N=\mathcal{F}_{\boldsymbol{\lambda},(\nu)}$ in the above, then it follows from (5.14) that

$$
\mathcal{\partial}_{\boldsymbol{\lambda}}=J_{>\nu} \oplus \bigoplus_{\boldsymbol{\lambda} \preceq \boldsymbol{\mu} \preceq(\nu)} \mathcal{J}_{\boldsymbol{\lambda}}^{\prime}
$$

for each $\boldsymbol{\lambda} \in \Lambda_{\nu}(\mathbb{N})$. The decomposition of $\mathcal{J}_{\boldsymbol{\lambda}}$ now follows by induction since $J_{>\nu}=J_{\left(\nu_{+}\right)}$, where $\nu_{+}$denotes an immediate successor of $\nu$ in the lexicographic order on $\Lambda_{d}(r)$. The decomposition for $\Gamma^{d} J=\mathcal{J}_{\left(1^{d}\right)}$ follows as a special case.

Now suppose $\boldsymbol{\lambda} \in \Lambda_{d}^{+}(\mathbb{N})^{r}$. To each element of the basis (5.13), we associate a corresponding element in $\mathcal{J}_{\boldsymbol{\lambda}}^{\prime}$, defined by

$$
z_{\mathbf{S}, \mathbf{T}}:=\left({ }^{\prime} \nabla^{\nu} \circ \Gamma^{(\nu)}\left(\alpha_{*}^{\prime}\right)^{-1} \circ \psi^{\boldsymbol{\lambda}}\right)\left(x_{\mathbf{S}} \otimes y_{\mathbf{T}}\right) .
$$

Since the map appearing in (5.20) is a composition of isomorphisms, it follows that the set

$$
\left\{z_{\mathbf{S}, \mathbf{T}} \mid \mathbf{S} \in \mathrm{St}_{\boldsymbol{\lambda}}\left(\mathcal{B}_{*}\right), \mathbf{T} \in \mathrm{St}_{\boldsymbol{\lambda}}\left(\mathcal{C}_{*}\right)\right\}
$$

forms a basis of $\mathcal{\partial}_{\boldsymbol{\lambda}}^{\prime}$.

Let $\left(m_{1}, \ldots, m_{r}\right) \in \mathbb{N}^{r}$ be the sequence defined by

$$
m_{j}:=\min \left(\sharp \mathcal{B}_{j}, \sharp \mathcal{C}_{j}\right)
$$

for all $j$, and set

$$
\boldsymbol{\Lambda}:=\Lambda_{r}^{+}\left(m_{1}, \ldots, m_{r}\right) .
$$

Remark 5.12. Suppose $\boldsymbol{\lambda} \in \Lambda^{+}(\mathbb{N})^{r}$. If $\boldsymbol{\lambda}$ belongs to $\boldsymbol{\Lambda} \subset \Lambda^{+}(\mathbb{N})^{r}$, then $\operatorname{St}\left(\mathcal{B}_{*}\right)$ and $\operatorname{St}\left(\mathcal{C}_{*}\right)$ are both non-empty since they contain the elements $\mathrm{T}^{\boldsymbol{\lambda}}=\mathrm{T}^{\boldsymbol{\lambda}}\left(\mathcal{B}_{*}\right)$ and $\mathrm{T}^{\boldsymbol{\lambda}}=\mathrm{T}^{\boldsymbol{\lambda}}\left(\mathcal{C}_{*}\right)$ defined in $(5.9)$, respectively. We thus have $\boldsymbol{\partial}_{\boldsymbol{\lambda}}^{\prime} \neq 0$ if and only if $\lambda \in \Lambda$.

Let $\boldsymbol{\lambda} \in \boldsymbol{\Lambda}$. Since $\mathcal{J}_{\boldsymbol{\lambda}}=\mathcal{J}_{\boldsymbol{\lambda}}^{\prime} \oplus \mathcal{J}_{\boldsymbol{\lambda}^{+}}$by Lemma 5.11 , it follows that $\mathcal{J}_{\boldsymbol{\lambda}} / \mathcal{J}_{\boldsymbol{\lambda}^{+}}$is a free $\mathbb{k}$-module with basis

$$
\left\{\bar{z}_{\mathbf{S}, \mathbf{T}} \mid \mathbf{S} \in \mathrm{St}_{\boldsymbol{\lambda}}\left(\mathcal{B}_{*}\right), \mathbf{T} \in \mathrm{St}_{\boldsymbol{\lambda}}\left(\mathcal{C}_{*}\right)\right\}
$$

where $\bar{x}:=x+\mathcal{J}_{\boldsymbol{\lambda}^{+}}$denotes the image of $x \in \mathcal{J}_{\boldsymbol{\lambda}}$ in the quotient.

Definition 5.13. Given $\boldsymbol{\lambda} \in \boldsymbol{\Lambda}$, define a pair of $\mathbb{k}$-submodules

$$
\mathcal{U}_{\boldsymbol{\lambda}}, \mathcal{V}_{\boldsymbol{\lambda}} \subset \mathcal{J}_{\boldsymbol{\lambda}} / \mathcal{J}_{\boldsymbol{\lambda}^{+}}
$$

generated by the subsets

$$
\left\{\bar{z}_{\mathbf{S}, \mathbf{T}^{\boldsymbol{\lambda}}} \mid \mathbf{S} \in \operatorname{St}_{\boldsymbol{\lambda}}\left(\mathcal{B}_{*}\right)\right\} \quad \text { and } \quad\left\{\bar{z}_{\mathbf{T}^{\boldsymbol{\lambda}}, \mathbf{T}} \mid \mathbf{T} \in \operatorname{St}_{\boldsymbol{\lambda}}\left(\mathcal{C}_{*}\right)\right\}
$$

respectively. It is then clear that $\mathcal{U}_{\boldsymbol{\lambda}}$ is a $\Gamma^{d} A$-submodule of the $\left(\Gamma^{d} A, \Gamma^{d} B\right)$ bimodule $\mathcal{J}_{\boldsymbol{\lambda}} / \mathcal{J}_{\boldsymbol{\lambda}^{+}}$, and $\mathcal{V}_{\boldsymbol{\lambda}}$ is a $\Gamma^{d} B$-submodule.

The following analogue of Theorem 5.4 is the main result in this section.

Theorem 5.14 (Generalized Cauchy Decomposition). Suppose $\boldsymbol{\lambda} \in \boldsymbol{\Lambda}$. Then the map of $\mathbb{k}$-modules defined by

$$
\alpha_{\boldsymbol{\lambda}}: \mathcal{J}_{\boldsymbol{\lambda}} / \mathcal{J}_{\boldsymbol{\lambda}^{+}} \rightarrow \mathcal{U}_{\boldsymbol{\lambda}} \otimes \mathcal{V}_{\boldsymbol{\lambda}}: \quad \bar{z}_{\mathbf{S}, \mathbf{T}} \mapsto \bar{z}_{\mathbf{S}, \mathbf{T}^{\boldsymbol{\lambda}}} \otimes \bar{z}_{\mathbf{T}^{\boldsymbol{\lambda}}, \mathbf{T}},
$$

for all $(\mathbf{S}, \mathbf{T}) \in \operatorname{St}_{\boldsymbol{\lambda}}\left(\mathcal{B}_{*}\right) \times \operatorname{St}_{\boldsymbol{\lambda}}\left(\mathcal{C}_{*}\right)$, is an isomorphism of $\left(\Gamma^{d} A, \Gamma^{d} B\right)$-bimodules. The associated graded module of the generalized Cauchy filtration is thus given by

$$
\bigoplus_{\lambda \in \Lambda} u_{\boldsymbol{\lambda}} \otimes v_{\lambda}
$$


Proof. Write $\phi_{\boldsymbol{\lambda}}: \mathcal{J}_{\boldsymbol{\lambda}} \rightarrow \mathcal{F}_{\boldsymbol{\lambda},(\nu)}$ to denote the map obtained from $\hat{\phi}_{\nu}$ by restriction. There is an induced bimodule isomorphism

$$
\bar{\phi}_{\boldsymbol{\lambda}}: \mathcal{J}_{\boldsymbol{\lambda}} / \mathcal{J}_{\boldsymbol{\lambda}^{+}} \stackrel{\sim}{\longrightarrow} \mathcal{F}_{\boldsymbol{\lambda},(\nu)} / \mathcal{F}_{\boldsymbol{\lambda}^{+},(\nu)}
$$

which follows from the definitions by using the decompositions $\mathcal{J}_{\boldsymbol{\lambda}}=\mathcal{J}_{\boldsymbol{\lambda}}^{\prime} \oplus \mathcal{J}_{\boldsymbol{\lambda}^{+}}$and $\mathcal{F}_{\boldsymbol{\lambda},(\nu)}=\mathcal{F}_{\boldsymbol{\lambda}}^{\prime} \oplus \mathcal{F}_{\boldsymbol{\lambda}^{+},(\nu)}$.

Hence by Proposition 5.9, there is an isomorphism $\varphi_{\boldsymbol{\lambda}}$ making the upper right triangle commute in the following diagram

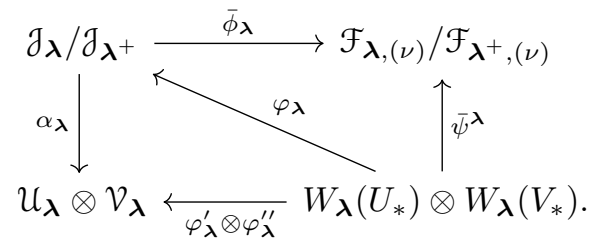

In the bottom arrow, the map $\varphi_{\boldsymbol{\lambda}}^{\prime}\left(\right.$ resp. $\left.\varphi_{\boldsymbol{\lambda}}^{\prime \prime}\right)$ denotes the homomorphism obtained by composing $\varphi_{\boldsymbol{\lambda}}$ with the embedding

$$
W_{\boldsymbol{\lambda}}\left(U_{*}\right) \stackrel{\sim}{\longrightarrow} W_{\boldsymbol{\lambda}}\left(U_{*}\right) \otimes \bar{y}_{\mathbf{T}^{\boldsymbol{\lambda}}} \quad\left(\text { resp. } W_{\boldsymbol{\lambda}}\left(V_{*}\right) \stackrel{\sim}{\sim} \bar{x}_{\mathbf{T}^{\boldsymbol{\lambda}}} \otimes W_{\boldsymbol{\lambda}}\left(V_{*}\right)\right) .
$$

In order to complete the proof, it suffices to show that the lower triangle in (5.21) is a commutative triangle of isomorphisms. For this, we compute:

$$
\begin{array}{rlr}
\varphi_{\boldsymbol{\lambda}}\left(\bar{x}_{\mathbf{S}} \otimes \bar{y}_{\mathbf{T}}\right) & =\left(\bar{\phi}_{\boldsymbol{\lambda}}^{-1} \circ \bar{\psi}^{\boldsymbol{\lambda}}\right)\left(\bar{x}_{\mathbf{S}} \otimes \bar{y}_{\mathbf{T}}\right) & \\
& =\bar{\phi}_{\boldsymbol{\lambda}}^{-1}\left(\overline{\psi^{\boldsymbol{\lambda}}\left(x_{\mathbf{S}} \otimes y_{\mathbf{T}}\right)}\right) & \text { by Prop. } 5.9 \\
& =\overline{\left(\phi_{\boldsymbol{\lambda}}^{\prime}\right)^{-1} \circ \psi^{\boldsymbol{\lambda}}\left(x_{\mathbf{S}} \otimes y_{\mathbf{T}}\right)} & \text { by (5.18) and }(5.19) \\
& =\bar{z}_{\mathbf{S}, \mathbf{T} .}
\end{array}
$$

It follows that $\varphi_{\boldsymbol{\lambda}}^{\prime} \otimes \varphi_{\boldsymbol{\lambda}}^{\prime \prime}$ is an isomorphism since

$$
\varphi_{\boldsymbol{\lambda}}^{\prime} \otimes \varphi_{\boldsymbol{\lambda}}^{\prime \prime}\left(\bar{x}_{\mathbf{S}} \otimes \bar{y}_{\mathbf{T}}\right)=\bar{z}_{\mathbf{S}, \mathbf{T}^{\boldsymbol{\lambda}}} \otimes \bar{z}_{\mathbf{T}^{\boldsymbol{\lambda}}, \mathbf{T}}
$$

for all $(\mathbf{S}, \mathbf{T}) \in \operatorname{St}_{\boldsymbol{\lambda}}\left(\mathcal{B}_{*}\right) \times \operatorname{St}_{\boldsymbol{\lambda}}\left(\mathcal{C}_{*}\right)$. Since it is now clear that the lower triangle is commutative, the proof is complete.

It follows from the proof of the theorem that $\mathcal{U}_{\boldsymbol{\lambda}}$ and $\mathcal{V}_{\boldsymbol{\lambda}}$ are each isomorphic to a respective (generalized) Weyl module. In the case $B=A^{\mathrm{op}}$, we call $\mathcal{U}_{\boldsymbol{\lambda}}$ (resp. $\mathcal{V}_{\boldsymbol{\lambda}}$ ) a left (resp. right) Weyl submodule of the $\Gamma^{d} A$-bimodule $\mathcal{J}_{\boldsymbol{\lambda}} / \mathcal{J}_{\boldsymbol{\lambda}^{+}}$.

\section{Cellular Algebras}

Assume throughout this section that $\mathbb{k}$ is a noetherian integral domain. We first recall the definition of cellular algebras from [8], along with the reformulation given in [16]. We then use the generalized Cauchy decomposition to describe a cellular structure on generalized Schur algebras $S^{A}(n, d)$.

\subsection{Definition of cellular algebras.}

Definition 6.1 (Graham-Lehrer). An associative $\mathbb{k}$-algebra $A$ is called a cellular algebra with cell datum $(I, M, C, \tau)$ if the following conditions are satisfied:

(C1) $(I, \unrhd)$ is a finite partially ordered set. Associated to each $\lambda \in I$ is a finite set $M(\lambda)$. The algebra $A$ has a $\mathbb{k}$-basis $C_{S, T}^{\lambda}$, where $(S, T)$ runs through all elements of $M(\lambda) \times M(\lambda)$ for all $\lambda \in I$.

(C2) The map $\tau$ is an anti-involution of $A$ such that $\tau\left(C_{S, T}^{\lambda}\right)=C_{T, S}^{\lambda}$. 
(C3) For each $\lambda \in I$ and $S, T \in M(\lambda)$ and each $a \in A$, the product $a C_{S, T}^{\lambda}$ can be written as $\left(\sum_{U \in M(\lambda)} r_{a}(U, S) C_{U, T}^{\lambda}\right)+r^{\prime}$, where $r^{\prime}$ is a linear combination of basis elements with upper index $\mu$ strictly larger than $\lambda$, and where the coefficients $r_{a}(U, S) \in \mathbb{k}$ do not depend on $T$.

Let $A$ be a cellular algebra with cell datum $(I, M, C, \tau)$. Given $\lambda \in I$, it is clear that the set $J(\lambda)$ spanned by the $C_{S, T}^{\mu}$ with $\mu \unrhd \lambda$ is a $\tau$-invariant two sided ideal of $A$ (see [8]). Let $J(\triangleright \lambda)$ denote the sum of ideals $J(\mu)$ with $\mu \triangleright \lambda$.

For $\lambda \in I$, the standard module $\Delta(\lambda)$ is defined as follows: as a $\mathbb{k}$-module, $\Delta(\lambda)$ is free with basis indexed by $M(\lambda)$, say $\left\{C_{S}^{\lambda} \mid S \in M(\lambda)\right\}$; for each $a \in A$, the action of $a$ on $\Delta(\lambda)$ is defined by $a C_{S}^{\lambda}=\sum_{U} r_{a}(U, S) C_{U}^{\lambda}$ where the elements $r_{a}(U, S) \in \mathbb{k}$ are the coefficients in (C3). Any left $A$-module isomorphic to $\Delta(\lambda)$ for some $\lambda$ will also be called a standard module. Note that for any $T \in M(\lambda)$, the assignment $C_{S}^{\lambda} \mapsto C_{S, T}^{\lambda}+J(\triangleright \lambda)$ defines an injective $A$-module homomorphism from $\Delta(\lambda)$ to $J(\lambda) / J(\triangleright \lambda)$.

6.2. Basis-free definition of cellular algebras. In [16], König and Xi provide an equivalent definition of cellular algebras which does not require specifying a particular basis. This definition can be formulated as follows.

Definition 6.2 (König-Xi). Suppose $A$ is a $\mathbb{k}$-algebra with an anti-involution $\tau$. Then a two-sided ideal $J$ in $A$ is called a cell ideal if, and only if, $J=\tau(J)$ and there exists a left ideal $\Delta \subset J$ such that $\Delta$ is finitely generated and free over $\mathbb{k}$ and such that there is an isomorphism of $A$-bimodules $\alpha: J \stackrel{\sim}{\longrightarrow} \Delta \otimes \tau(\Delta)$ making the following diagram commutative:

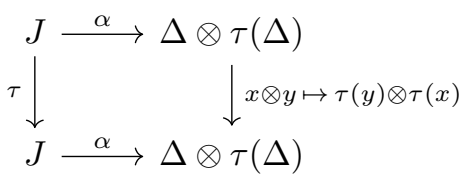

We say that a decomposition $A=J_{1}^{\prime} \oplus \cdots \oplus J_{r}^{\prime}$ (for some $r$ ) into $\mathbb{k}$-submodules with $\tau\left(J_{j}^{\prime}\right)=J_{j}^{\prime}$ for each $j=1, \ldots, r$ is a cellular decomposition of $A$ if setting $J_{j}:=\bigoplus_{1 \leq i \leq j} J_{i}^{\prime}$ gives a chain of ( $\tau$-invariant) two-sided ideals

$$
0=J_{0} \subset J_{1} \subset J_{2} \subset \cdots \subset J_{r}=A
$$

such that the quotient $J_{j} / J_{j-1}$ is a cell ideal (with respect to the anti-involution induced by $\tau$ on the quotient) of $A / J_{j-1}$.

The above chain of ideals in $A$ is called a cell chain. For each ideal $J_{j}$ in a cell chain, we write

$$
\Delta_{j} \subset J_{j} / J_{j-1}, \quad \alpha_{j}: J_{j} / J_{j-1} \stackrel{\sim}{\longrightarrow} \Delta_{j} \otimes \tau\left(\Delta_{j}\right)
$$

to denote the corresponding left ideal and $A$-bimodule isomorphism. Since $J_{j}=$ $J_{j}^{\prime} \oplus J_{j-1}$ for all $j$, we have a $\mathbb{k}$-module isomorphism $\alpha_{j}^{\prime}: J_{j}^{\prime} \cong \Delta_{j} \otimes \tau\left(\Delta_{j}\right)$ defined as the composition

$$
\alpha_{j}^{\prime}: J_{j}^{\prime} \hookrightarrow J_{j} \rightarrow J_{j} / J_{j-1} \stackrel{\alpha_{j}}{\longrightarrow} \Delta_{j} \otimes \tau\left(\Delta_{j}\right)
$$

It then follows by definition that we have a commutative diagram

$$
\begin{aligned}
& J_{j}^{\prime} \stackrel{\alpha_{j}^{\prime}}{\longrightarrow} \Delta_{j} \otimes_{\mathbb{k}} \tau\left(\Delta_{j}\right) \\
& i \downarrow \\
& \downarrow \\
& J_{j}^{\prime} \stackrel{\alpha_{j}^{\prime}}{\longrightarrow} \Delta_{j} \otimes_{\mathbb{k}} \tau\left(\Delta_{j}\right)
\end{aligned}
$$

of $\mathbb{k}$-module isomorphisms. 
Lemma 6.3 (König-Xi, [16]). Let $A$ be an associative $\mathbb{k}$-algebra with an antiinvolution $\tau$. Then $A$ is a cellular algebra in the sense of [8] if and only if $A$ has a cellular decomposition.

Proof. We summarize the proof from [16]. Let $A$ be a cellular algebra with cell datum $(I, M, C, \tau)$. First, suppose $\lambda \in I$ is maximal. Then $J=J(\lambda)$ is a two-sided ideal by (C3) and $J=\tau(J)$ by (C2). Fix any element $T_{\lambda} \in M(\lambda)$. Define $\Delta$ as the $\mathbb{k}$-span of $C_{S, T_{\lambda}}^{\lambda}$ where $S$ varies. Defining $\alpha$ by sending $C_{S, T_{\lambda}}^{\lambda} \otimes \tau\left(C_{T, T_{\lambda}}^{\lambda}\right)$ to $C_{S, T}^{\lambda}$ gives the required isomorphism. Thus $J(\lambda)$ is a cell ideal.

Next, choose any enumeration $\lambda_{1}, \ldots, \lambda_{r}$ of the elements of $I$ such that $i<j$ whenever $\lambda_{j} \triangleright \lambda_{i}$. Set $J_{j}^{\prime} \subset A$ (for each $j$ ) equal to the $\mathbb{k}$-span of all $C_{S, T}^{\lambda_{j}}$ (for varying $S, T)$. We have $\tau\left(J_{j}^{\prime}\right)=J_{j}^{\prime}$ by $(\mathrm{C} 2)$. Since $J\left(\lambda_{j}\right)=J_{j}^{\prime} \bigoplus J\left(\triangleright \lambda_{j}\right)$ for all $j$, it follows that $A=\bigoplus_{j} J_{j}^{\prime}$ is a cellular decomposition.

For the converse, consider the index set $I=\{1, \ldots, r\}$ with the reversed ordering $1 \triangleright \cdots \triangleright r$. Choose a $\mathbb{k}$-basis $\left\{x_{b}^{(j)}\right\}_{b \in \mathcal{B}_{j}}$ of $\Delta_{j}$, for each $j \in I$. Setting $C_{b, c}^{j} \in J_{j}^{\prime}$ to be the inverse image of $x_{b}^{(j)} \otimes \tau\left(x_{c}^{(j)}\right.$ ) (for $b, c \in \mathcal{B}_{j}$ ) under $\alpha_{j}^{\prime}$ (for $j \in I$ ) gives a $\mathbb{k}$-basis for $A$ of the form (C1). Since $\Delta_{j}$ is a left $A$-module, (C3) is satisfied. Finally, (C2) follows from the required commutative diagram and the $\tau$-invariance of $J_{j}^{\prime}$. It follows that $\left\{C_{b, c}^{j}\right\}$ is a cellular basis.

From now on, we say that an algebra $A$ with anti-involution $\tau$ is cellular if either of the equivalent statements in Lemma 6.3 is satisfied. The proof of the lemma shows that each ideal $\Delta_{j}$ (for $j=1, \ldots, r$ ) for a cellular algebra $A$ is a standard module.

6.3. Matrix algebras. Consider the matrix ring, $\mathrm{M}_{n}(\mathbb{k})$, with matrix transpose, tr, as anti-involution. Let us write, $c: \mathrm{V}_{n} \otimes \mathrm{V}_{n}^{\mathrm{tr}} \stackrel{\sim}{\longrightarrow} \mathrm{M}_{n}(\mathbb{k})$, to denote the isomorphism mapping $v_{i} \otimes v_{j}^{\text {tr }} \mapsto E_{i j}$ for all $i, j \in[1, n]$.

Now suppose $A$ is an algebra with anti-involution $\tau$, and let $J$ be a cell ideal with defining isomorphism $\alpha: J \stackrel{\sim}{\longrightarrow} \Delta \otimes \tau(\Delta)$. Then

$$
\mathrm{M}_{n}(J):=\mathrm{M}_{n}(\mathbb{k}) \otimes J
$$

is a cell ideal of the matrix ring $\mathrm{M}_{n}(A)$ with respect to the anti-involution $\operatorname{tr} \otimes \tau$. The corresponding isomorphism is the map

$$
c^{-1}(\alpha): \mathrm{M}_{n}(J) \stackrel{\sim}{\longrightarrow} \mathrm{V}_{n}(\Delta) \otimes \mathrm{V}_{n}^{\mathrm{tr}}(\tau(\Delta))
$$

defined by the composition

$$
\mathrm{M}_{n}(\mathbb{k}) \otimes J \stackrel{c^{-1} \otimes \alpha}{\longrightarrow}\left(\mathrm{V}_{n} \otimes \mathrm{V}_{n}^{\mathrm{tr}}\right) \otimes(\Delta \otimes \tau(\Delta)) \stackrel{\sim}{\longrightarrow} \mathrm{V}_{n} \otimes \Delta \otimes \mathrm{V}_{n}^{\mathrm{tr}} \otimes \tau(\Delta) .
$$

More generally, we have the following.

Lemma 6.4. Suppose $A$ is a cellular algebra with anti-involution $\tau$ and cell chain $\left(J_{j}\right)_{j \in[1, r]}$. Then the matrix ring $\mathrm{M}_{n}(A)$ is cellular with anti-involution $\operatorname{tr} \otimes \tau$ and cell chain $\left(\mathrm{M}_{n}\left(J_{j}\right)\right)_{j \in[1, r]}$, where $\mathrm{M}_{n}\left(J_{j}\right):=\mathrm{M}_{n}(\mathbb{k}) \otimes J_{j}$ for all $j$.

Proof. It follows from the preceding paragraph that the ideals, $\mathrm{M}_{n}\left(J_{j}\right)$, form a cell chain, since $\mathrm{M}_{n}\left(J_{j}\right) / \mathrm{M}_{n}\left(J_{j-1}\right) \simeq \mathrm{M}_{n}(\mathbb{k}) \otimes\left(J_{j} / J_{j-1}\right)$ as $\mathrm{M}_{n}(A)$-bimodules. It is also clear that $\mathrm{M}_{n}(A)$ has a cellular decomposition

$$
\mathrm{M}_{n}(A)=\bigoplus \mathrm{M}_{n}\left(J_{j}^{\prime}\right)
$$

where $A=\bigoplus J_{j}^{\prime}$ denotes a corresponding cellular decomposition of $A$. 
6.4. Cellularity of generalized Schur algebras. We now describe a cellular structure for generalized Schur algebras $S^{A}(n, d)$. In this case, the generalized Cauchy filtration forms a cell chain, with the Weyl submodules from Theorem 5.14 as standard modules.

Theorem 6.5. Suppose $A$ is a cellular algebra with anti-involution $\tau$. Then the generalized Schur algebra $S^{A}(n, d)$ is a cellular algebra, with respect to the antiinvolution $\tau:=(\operatorname{tr} \otimes \tau)^{\otimes d}$, for all $n, d \in \mathbb{N}$.

Proof. If $A$ is cellular then so is $\mathrm{M}_{n}(A)$, by Lemma 6.4. Since $S^{A}(n, d)=\Gamma^{d} \mathrm{M}_{n}(A)$, it suffices to show that $\Gamma^{d} A$ is cellular, with respect to the anti-involution $\boldsymbol{\tau}=\tau^{\otimes d}$.

Suppose that $A=J_{1}^{\prime} \oplus \cdots \oplus J_{r}^{\prime}$ is a cellular decomposition of $A$, with corresponding cell chain

$$
0=J_{0} \subset J_{1} \subset \cdots \subset J_{r}=A \text {. }
$$

For each $j \in[1, r]$, suppose $\left\{x_{b}^{(j)}\right\}_{b \in \mathcal{B}_{j}}$ and $\left\{y_{b}^{(j)}\right\}_{b \in \mathcal{B}_{j}}$ are $\mathbb{k}$-bases of $\Delta_{j}$ and $\tau\left(\Delta_{j}\right)$, respectively, such that $y_{b}^{(j)}:=\tau\left(x_{b}^{(j)}\right)$ for all $j$, and let $\Delta_{j}$ and $\alpha_{j}$ be as in (6.1).

Considering $\boldsymbol{\Lambda}=\Lambda^{+}\left(\sharp \mathcal{B}_{1}, \ldots, \sharp \mathcal{B}_{r}\right)$ as a totally ordered subset of $\Lambda_{d}^{+}(\mathbb{N})^{r}$ by restricting the order $\preceq$ in Definition 2.4, it follows from Lemma 5.11 and Remark 5.12 that we have decompositions

$$
\Gamma^{d} J=\bigoplus_{\boldsymbol{\lambda} \in \boldsymbol{\Lambda}} \mathcal{J}_{\boldsymbol{\lambda}}^{\prime}, \quad \text { and } \quad \partial_{\boldsymbol{\lambda}}=\bigoplus_{\boldsymbol{\mu} \succeq \boldsymbol{\lambda}} \mathcal{J}_{\boldsymbol{\mu}}^{\prime} \quad \text { for each } \boldsymbol{\lambda} \in \boldsymbol{\Lambda}
$$

since $\partial_{\boldsymbol{\lambda}}^{\prime}=0$ if $\boldsymbol{\lambda} \notin \boldsymbol{\Lambda}$.

Notice that $\tau=\tau^{\otimes d}$ coincides with the map $\Gamma^{d}(\tau): \Gamma^{d} A \rightarrow \Gamma^{d} A$ induced by the functor $\Gamma^{d}$. To complete the proof, we need to show that the left-hand side of (6.3) gives a cellular decomposition of $\Gamma^{d} A$ with respect to this anti-involution.

Let $\Delta_{\boldsymbol{\lambda}}$ be the the left Weyl submodule $\mathcal{U}_{\boldsymbol{\lambda}} \subset \mathcal{J}_{\boldsymbol{\lambda}} / \mathcal{J}_{\boldsymbol{\lambda}^{+}}$of Theorem 5.14. Then it remains to check the following hold for each $\boldsymbol{\lambda} \in \boldsymbol{\Lambda}$ :

(i) $\boldsymbol{\tau}\left(\partial_{\boldsymbol{\lambda}}^{\prime}\right)=\mathcal{J}_{\boldsymbol{\lambda}}^{\prime}$

(ii) $\left.\tau_{\left(\Delta_{\boldsymbol{\lambda}}\right.}\right)=\mathcal{V}_{\boldsymbol{\lambda}}$,

(iii) $\mathcal{J}_{\boldsymbol{\lambda}} / \mathcal{\partial}_{\boldsymbol{\lambda}^{+}}$is a cell ideal.

Assuming (i) and (ii) hold for each $\boldsymbol{\lambda}$, (iii) will follow from the commutativity of the diagram

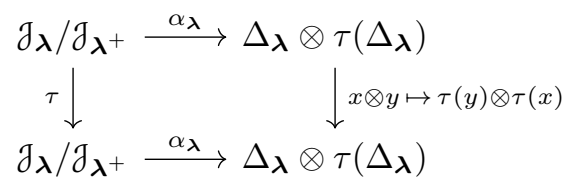

where $\alpha_{\boldsymbol{\lambda}}$ is the $\Gamma^{d} A$-bimodule isomorphism from Theorem 5.14.

Now fix $\boldsymbol{\lambda} \in \boldsymbol{\Lambda}$, and set $\nu=|\boldsymbol{\lambda}|$. Then $\mathcal{J}_{\boldsymbol{\lambda}}^{\prime}, \Delta_{\boldsymbol{\lambda}}$, and $\mathcal{J}_{\boldsymbol{\lambda}} / \mathcal{J}_{\boldsymbol{\lambda}^{+}}$have $\mathbb{k}$-bases given by the sets

$$
\left\{z_{\mathbf{S}, \mathbf{T}} \mid \mathbf{S}, \mathbf{T} \in \operatorname{St}\left(\mathcal{B}_{*}\right)\right\}, \quad\left\{\bar{z}_{\mathbf{S}, \mathbf{T}_{\boldsymbol{\lambda}}} \mid \mathbf{S} \in \operatorname{St}\left(\mathcal{B}_{*}\right)\right\}, \quad\left\{\bar{z}_{\mathbf{S}, \mathbf{T}} \mid \mathbf{S}, \mathbf{T} \in \operatorname{St}\left(\mathcal{B}_{*}\right)\right\}
$$

respectively, where $z_{\mathbf{S}, \mathbf{T}} \in \mathcal{J}_{\boldsymbol{\lambda}}^{\prime}$ is defined in (5.20). It follows that each of the conditions (i)-(iii) will be satisfied provided that $\tau\left(z_{\mathbf{S}, \mathbf{T}}\right)=z_{\mathbf{T}, \mathbf{S}}$ for all $\mathbf{S}, \mathbf{T} \in$ $\operatorname{St}\left(\mathcal{B}_{*}\right)$. 
We claim that the following diagram is commutative:

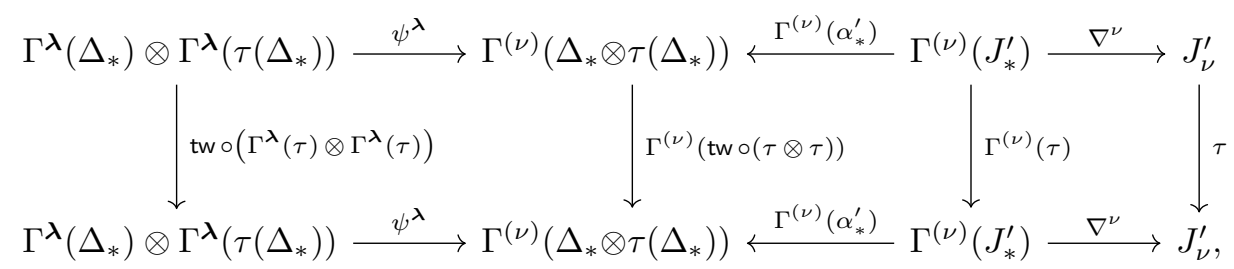

with the first (middle) vertical map(s) induced by the action of $\Gamma^{\boldsymbol{\lambda}}$ (resp. $\Gamma^{(\nu)}$ ) considered as a functor $\mathcal{P}_{\mathbb{k}}^{\times r} \rightarrow \mathcal{P}_{\mathbb{k}}$. The commutativity of the left-hand square can be checked using the definition of $\psi^{\boldsymbol{\lambda}}$ together with Lemma 2.5. The commutativity of the middle square follows from the functoriality of $\Gamma^{(\nu)}$ and diagram (6.2). Finally, the commutativity of the right-hand square follows by Lemma 3.4. We thus have $\tau\left(z_{\mathbf{S}, \mathbf{T}}\right)=z_{\mathbf{T}, \mathbf{S}}$ for all $\mathbf{S}, \mathbf{T} \in \mathbf{S t}\left(\mathcal{B}_{*}\right)$, and the proof is complete.

Let us write $\boldsymbol{\Lambda}^{\mathrm{op}}$ to denote the set $\boldsymbol{\Lambda}$ with opposite total ordering. Then it follows from the above proofs of Lemma 6.3 and Theorem 6.5 that the set

$$
\left\{z_{\mathbf{S}, \mathbf{T}} \mid \boldsymbol{\lambda} \in \boldsymbol{\Lambda}^{\mathrm{op}}, \mathbf{S}, \mathbf{T} \in \mathrm{St}_{\boldsymbol{\lambda}}\left(\mathcal{B}_{1}, \ldots, \mathcal{B}_{r}\right)\right\} .
$$

is a cellular basis for $\Gamma^{d} A$. A corresponding cellular basis for $S^{A}(n, d)$ can be obtained in a similar way, by replacing $A$ by $\mathrm{M}_{n}(A)$.

In the next example, we describe an explicit cellular basis for a special case of a generalized Schur algebra of the form $S^{Z}(n, d)$, where $Z$ is a zig-zag algebra. We essentially follow the definition in [15], using slightly different notation. Note also that we only consider $Z$ as an ordinary non-graded algebra, rather than a $\mathbb{Z} / 2$-graded superalgebra as in $[15]$.

Example 6.6 (Zig-zag algebra). We consider the zig-zag algebra associated to the quiver below.

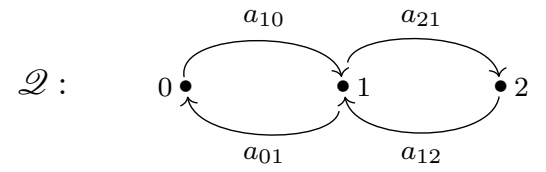

Recall from [15, Section 7.9] that the extended zig-zag algebra, $\tilde{Z}$, is defined in this case as the quotient of the path algebra $\mathbb{k} \mathscr{Q}$ modulo the following relations:

(1) All paths of length three or greater are zero.

(2) All paths of length two that are not cycles are zero.

(3) All length-two cycles based at the same vertex are equivalent.

(4) $a_{21} a_{12}=0$.

The length zero paths are denoted $e_{0}, e_{1}, e_{2}$ and correspond to standard idempotents, with $e_{i} a_{i j} e_{j}=a_{i j}$ for all admissible $i, j$. Let $e:=e_{0}+e_{1} \in \tilde{Z}$. Then the corresponding zig-zag algebra is $Z:=e \tilde{Z} e \subset \tilde{Z}$. Then $Z$ is a cellular algebra, with anti-involution defined by $\tau\left(e_{i}\right)=e_{i}$ and $\tau\left(a_{i j}\right)=a_{j i}$ for all $i, j$.

Let us describe a corresponding cellular decomposition. First let

$$
x_{1}:=a_{12}, \quad x_{2}:=e_{1}, \quad x_{3}:=a_{01}, \quad x_{4}:=e_{0} .
$$

and set $y_{i}:=\tau\left(x_{i}\right)$, for $i \in[1,4]$. Then we have corresponding sets

$$
X(1):=\left\{x_{1}\right\}, \quad X(2):=\left\{x_{2}, x_{3}\right\}, \quad X(3):=\left\{x_{4}\right\},
$$

and

$$
Y(1):=\left\{y_{1}\right\}, \quad Y(2):=\left\{y_{2}, y_{3}\right\}, \quad Y(3):=\left\{y_{4}\right\}
$$


parametrized by the totally ordered sets $\mathcal{B}_{1}:=\{1\}, \mathcal{B}_{2}:=\{2<3\}$, and $\mathcal{B}_{3}:=\{4\}$, respectively. We may then define a cellular decomposition

$$
Z=J_{1}^{\prime} \oplus J_{2}^{\prime} \oplus J_{3}^{\prime},
$$

where $J_{j}^{\prime}:=\operatorname{span}\{x y \mid x \in X(j), y \in Y(j)\}$, for $j \in[1,3]$.

Now let $\boldsymbol{\Lambda}^{\text {op }}$ denote the set $\boldsymbol{\Lambda}=\Lambda_{3}^{+}(1,2,1)$ with the opposite total ordering. Then one may then check using formula (5.20) and the proof of Lemma 6.3 that $S^{Z}(1,2)=\Gamma^{2} Z$ has the cellular basis described in the table below, where $\boldsymbol{\lambda}$ runs through all multipartitions in the set $\boldsymbol{\Lambda}^{\mathrm{op}}$, and where $\mathbf{S}, \mathbf{T}$ denote standard multitableaux of shape $\boldsymbol{\lambda}$, respectively.

\begin{tabular}{|c|c|c|c|}
\hline$\lambda$ & $\mathbf{S}$ & $\mathbf{T}$ & $z_{\mathbf{S}, \mathbf{T}}$ \\
\hline$(\varnothing, \varnothing,(2))$ & $(\varnothing, \varnothing, 4[4)$ & $(\varnothing, \varnothing, 4,4)$ & $e_{0}^{\otimes 2}$ \\
\hline$(\varnothing,(1),(1))$ & $\begin{array}{c}(\varnothing, 2,4) \\
\prime \prime \\
(\varnothing, 3,4) \\
\quad, \quad 4\end{array}$ & $\begin{array}{l}(\varnothing, 2,4) \\
(\varnothing, 3,4) \\
(\varnothing, 2,4) \\
(\varnothing, 3,4)\end{array}$ & $\begin{array}{c}e_{0} * e_{1} \\
e_{0} * a_{10} \\
e_{0} * a_{01} \\
e_{0} *\left(a_{01} a_{10}\right)\end{array}$ \\
\hline$(\varnothing,(1,1), \varnothing)$ & $\left(\varnothing, \frac{2}{3}, \varnothing\right)$ & $\left(\varnothing, \frac{2}{3}, \varnothing\right)$ & $e_{1} *\left(a_{01} a_{10}\right)$ \\
\hline$(\varnothing,(2), \varnothing)$ & 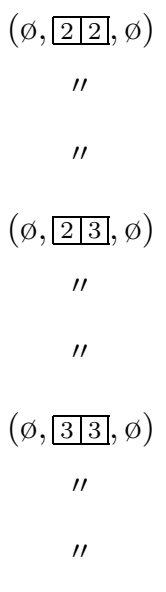 & 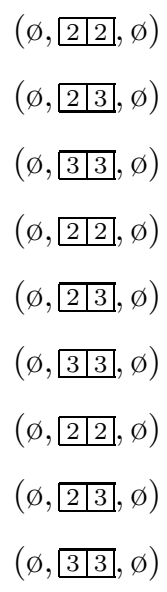 & $\begin{array}{c}e_{1}^{\otimes 2} \\
e_{1} * a_{10} \\
a_{10}^{\otimes 2} \\
e_{1} * a_{01} \\
e_{1} *\left(a_{01} a_{10}\right)+a_{10} * a_{01} \\
a_{10} * a_{01} a_{10} \\
a_{01}^{\otimes 2} \\
a_{01} *\left(a_{01} a_{10}\right) \\
\left(a_{01} a_{10}\right)^{\otimes 2}\end{array}$ \\
\hline$((1), \varnothing,(1))$ & $(4, \varnothing, 4)$ & $(1, \varnothing, 4)$ & $\left(a_{12} a_{21}\right) * e_{0}$ \\
\hline$((1),(1), \varnothing)$ & $\begin{array}{c}(1,, 2, \varnothing) \\
\prime \prime \\
(1,, 3, \varnothing) \\
\quad,\end{array}$ & $\begin{array}{l}(1, \sqrt{2}, \varnothing) \\
(1, \sqrt{3}, \varnothing) \\
(1,2, \varnothing) \\
(1,3, \varnothing)\end{array}$ & $\begin{array}{c}e_{1} *\left(a_{12} a_{21}\right) \\
a_{10} *\left(a_{12} a_{21}\right) \\
a_{01} *\left(a_{12} a_{21}\right) \\
\left(a_{01} a_{10}\right) *\left(a_{12} a_{21}\right)\end{array}$ \\
\hline$((2), \varnothing, \varnothing)$ & $\left(\begin{array}{l|l}1 & 1\end{array}, \varnothing, \varnothing\right)$ & $\left(\begin{array}{lll}1 & 1\end{array}, \varnothing, \varnothing\right)$ & $\left(a_{12} a_{21}\right)^{\otimes 2}$ \\
\hline
\end{tabular}

The symbol, $\varnothing$, is used above to denote an empty partition or tableau, respectively, and the symbol " denotes a repeated item from the above entry. 
6.5. Cellularity of wreath products $A$ < $\mathfrak{S}_{d}$. Let us first recall a result of [16] concerning idempotents fixed by an anti-involution.

Lemma 6.7 ([16]). Let $A$ be a cellular algebra with anti-involution $\tau$. If $e \in A$ is an idempotent fixed by $\tau$, then the algebra eAe is cellular with respect to the restriction of $\tau$.

We then have the following consequence of Theorem 6.5, which is obtained via generalized Schur-Weyl duality.

Corollary 6.8. Suppose $d \in \mathbb{N}$. If $A$ is a cellular algebra, then $A \nmid \mathfrak{S}_{d}$ is also cellular.

Proof. Fix some $n \geq d$. Write $S^{A}=S^{A}(n, d)$, and let $e \in S^{A}$ denote the idempotent $e:=\xi_{\omega}$. It then follows by Proposition 4.2.(ii) that there is an algebra isomorphism $A<\mathfrak{S}_{d} \cong e S^{A} e$. Since

$$
\boldsymbol{\tau}(e)=\left(E_{1,1}\right)^{\mathrm{tr}} * \cdots *\left(E_{d, d}\right)^{\mathrm{tr}}=e,
$$

the cellularity of $A<\mathfrak{S}_{d}$ follows from Theorem 6.5 and Lemma 6.7.

Since the above result holds for an arbitrary cellular algebra $A$, we thus obtain an alternate proof of the main results of [7] and [11] mentioned in the introduction.

\section{REFERENCES}

[1] K. Akin, D.A. Buchsbaum and J. Weyman, Schur functors and Schur complexes, Adv. in Math. 44 (1982), no. 3, 207-278.

[2] A.-L. Cauchy, Mémoire sur les fonctions alternées et sur les sommes alternées, Exercices d'analyse et de phys. math., ii (1841), 151-159; or Euvres complètes, 2ème série xii, GauthierVillars, Paris, 1916, 173-182.

[3] E. Cline, B. Parshall and L. Scott, Finite-dimensional algebras and highest weight categories, J. Reine Angew. Math. 391 (1988), 85-99.

[4] A. Evseev and A. Kleshchev, Turner doubles and generalized Schur algebras, Adv. Math. 317 (2017), 665-717.

[5] Blocks of symmetric groups, semicuspidal KLR algebras and zigzag Schur-Weyl duality, Ann. of Math. (2) $\mathbf{1 8 8}$ (2018), no. 2, 453-512.

[6] E.M. Friedlander and A. Suslin, Cohomology of finite group schemes over a field, Invent. Math. 127 (1997), no. 2, 209-270.

[7] T. Geetha and F.M. Goodman, Cellularity of wreath product algebras and A-Brauer algebras, J. Algebra 389 (2013), 151-190.

[8] J.J. Graham and G.I. Lehrer, Cellular algebras, Invent. Math. 123 (1996), 1-34.

[9] J.A. Green, Polynomial Representations of $G L_{n}$. (Lecture Notes in Math. 830), SpringerVerlag, New York 1980.

[10] _ Combinatorics and the Schur algebra, J. Pure Appl. Algebra 88 (1993), 89-106.

[11] R. Green, Cellular Structure of Wreath Product Algebras, J. Pure Appl. Algebra 224 (2020), no. 2, 819835 .

[12] M. Hashimoto and K. Kurano, Resolutions of determinantal ideals: $n$-minors of $(n+2)$-square matrices, Adv. Math. 94 (1992), no. 1, 1-66.

[13] A. Kleshchev and R. Muth, Based quasi-hereditary algebras, arXiv:1810.02844.

[14] - Generalized Schur algebras, arXiv:1810.02846.

[15] _ Schurifying quasi-hereditary algebras, arXiv:1810.02849.

[16] S. König, and C.C. Xi, On the structure of cellular algebras, Algebras and modules, II (Geiranger, 1996), 365-386, CMS Conf. Proc., 24, Amer. Math. Soc., Providence, RI, 1998.

[17] H. Krause, Highest weight categories and strict polynomial functors. With an appendix by Cosima Aquilino, EMS Ser. Congr. Rep., Representation theory, current trends and perspectives, 331-373, Eur. Math. Soc., Zürich, 2017.

[18] I.G. Macdonald, Symmetric functions and Hall polynomials, second edition, Oxford Math. Mon., (1995).

Sungkyunkwan University, Suwon 16419, Republic of Korea

E-mail address: jaxtell@skku.edu 